\title{
A general framework for solving Riemann-Hilbert problems numerically
}

\author{
Sheehan Olver
}

\begin{abstract}
A new, numerical framework for the approximation of solutions to matrix-valued Riemann-Hilbert problems is developed, based on a recent method for the homogeneous Painlevé II Riemann-Hilbert problem. We demonstrate its effectiveness by computing solutions to other Painlevé transcendents. An implementation in MAтнемAтіcA is made available online.
\end{abstract}

Keywords Riemann-Hilbert problems, singular integral equations, collocation methods, Painlevé transcendents.

Oxford University Mathematical Institute

Numerical Analysis Group

24-29 St Giles'

Oxford, England OX1 3LB

E-mail: Sheehan.0lver@sjc.ox.ac.uk

June, 2010 


\section{Introduction}

The solution to integrable, nonlinear differential equations can typically be written as Riemann-Hilbert (RH) problems. Examples include the Painlevé I-V transcendents [8], the Korteweg-de Vries (KdV) equation and the nonlinear Schrödinger (NLS) equation [1]. The importance of RH problems lies in the fact that they are often solvable asymptotically, which allows one to determine the asymptotics of solutions to the corresponding differential equation. This is accomplished using nonlinear steepest descent [5], a modification of a tool which is very familiar in asymptotic analysis: the method of steepest descent. In short, they can be viewed roughly as generalizations of integral representations.

RH problems also play an increasingly crucial role in the theory of orthogonal polynomials and random matrix theory [4]. Just as in the theory of integrable systems, they are useful in the determination of asymptotics of orthogonal polynomials. But the behaviour of orthogonal polynomials is in turn directly related to the asymptotics of eigenvalues of large random matrices. This has led to new results of universality: random matrices with vastly differing behaviour have eigenvalue distributions which are the same as the dimension of the matrix becomes large.

The reduction of a problem (such as a differential equation) to an integral representation is traditionally viewed as "solving" the problem, precisely because asymptotics are then readily available. But there is another important reason why integral representations are considered solutions: they can be used for numerics as well, through quadrature. The goal of this paper is to show that RH problems can also be used for numerics. Thus, reduction of a problem to a $\mathrm{RH}$ problem can be viewed, in a concrete sense, as a solution to the problem.

Though much research exists on numerical solution of the nonlinear RH problems used in conformal mapping $[\mathbf{1 9}, \mathbf{1 8}, \mathbf{2 0}, \mathbf{1 1}]$; the same is not true for the matrix-valued $\mathrm{RH}$ problems that are prevalent in modern applied analysis. The first use of matrix-valued RH problems as a numerical tool appears to be the thesis of Dienstfrey [6], which developed an approach for the computation of the RH problem connected with the sine kernel Fredholm determinant, of importance in random matrix theory. However, in this approach, an exponential amount of work at junction points of the domain was needed to achieve convergence, due to blow-up of the approximate solution at these points. This phenomenon was avoided in the development of an approach for the RH problem related to the homogeneous Painlevé II equation [14], by taking the behaviour at the junction point into account.

We will generalize the latter approach in the construction of a framework applicable to a broad class of $\mathrm{RH}$ problems. Moreover, it could potentially provide the foundation of a toolbox for computing Painlevé transcendents reliably. Just as RH problems are the nonlinear analogue of integral representations, Painlevé transcendents can be viewed as nonlinear special functions. 


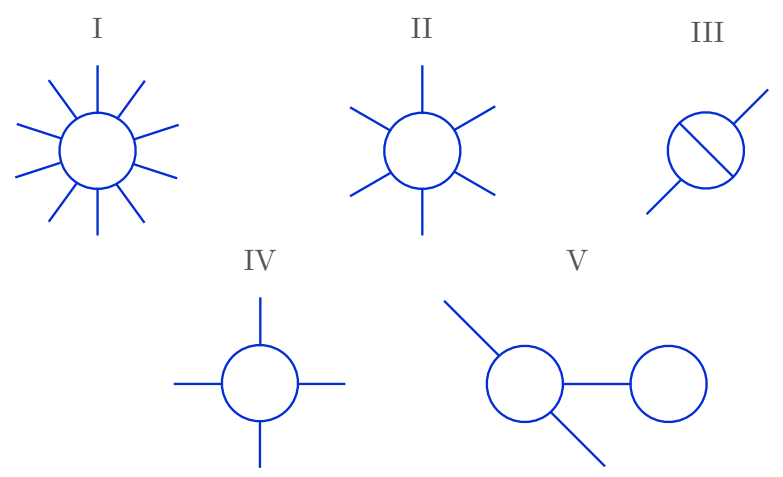

Figure 1: The jump curves for the first five Painlevé transcendents.

\section{Overview of the approach}

A RH problem is a boundary value problem of finding an analytic function which satisfies a given jump condition along a curve on which analyticity is lost. More precisely:

Problem 2.1 $[\mathbf{1 0}]$ Given an oriented curve $\Gamma \subset \mathbb{C}$ and a jump matrix $G: \Gamma \rightarrow \mathbb{C}^{2 \times 2}$, find a function $\Phi: \mathbb{C} \backslash \Gamma \rightarrow \mathbb{C}^{2 \times 2}$ which is analytic everywhere except on $\Gamma$ such that

$$
\begin{aligned}
& \Phi^{+}(z)=\Phi^{-}(z) G(z) \quad \text { for } z \in \Gamma \text { and } \\
& \Phi(\infty)=I
\end{aligned}
$$

where $\Phi^{+}$denotes the limit of $\Phi$ as $z$ approaches $\Gamma$ from the left, and $\Phi^{-}$denotes the limit of $\Phi$ as $z$ approaches $\Gamma$ from the right and $\Phi(\infty)=\lim _{|z| \rightarrow \infty} \Phi(z)$.

The jump curves $\Gamma$ for the first five Painlevé transcendents are depicted in Figure 1, cf. [8]. Note that each jump curve consists of a union of pieces conformally mappable to the unit interval: intervals, rays and arcs.

Consider the Cauchy transform (which is related to the Hilbert transform and Stieltjes transform)

$$
\mathcal{C}_{\Gamma} f(z)=\frac{1}{2 \mathrm{i} \pi} \int_{\Gamma} \frac{f(t)}{t-z} \mathrm{~d} t
$$

Normally, $\Gamma$ is implied by $f$, so we suppress the dependence and use simply $\mathcal{C}$. This operator maps a Hölder-continuous function $f$ defined on $\Gamma$ to a function which is analytic everywhere in the complex plane except on $\Gamma$, and which vanishes at $\infty$. Thus, as in $[6,14]$, we write $\Phi=\mathcal{C} U+I$, and Problem 2.1 becomes

$$
\mathcal{C}^{+} U-\left(\mathcal{C}^{-} U\right) G=G-I \quad \text { on } \Gamma
$$

where $\mathcal{C}^{ \pm}$denotes the left/right limits of the Cauchy transform. Now the operator $\mathcal{L} U=$ $\mathcal{C}^{+} U+\left(\mathcal{C}^{-} U\right) G$ is a linear map from the class of Hölder-continuous functions on $\Gamma$ to itself. 
We can use this linear operator in the construction of a collocation method. Suppose we have a sequence of points $\boldsymbol{x}=\left(x_{1}, \ldots, x_{N}\right)^{\top}$ on $\Gamma$ and we wish to represent the solution by its values at these points $\boldsymbol{U}=\left(U_{1}, \ldots, U_{N}\right)^{\top}\left(U_{i}\right.$ are either matrices or row vectors, but we treat them as elements in an abstract field, though what follows could be rewritten in terms of block matrices). Now if we have a scalar basis (represented as a row-vector) $\Psi(z)=\left(\psi_{1}(z), \ldots, \psi_{N}(z)\right)$ and a transform matrix $\mathcal{F}$ from values at $\boldsymbol{x}$ to the coefficients of $\Psi$, then, at least conceptually, we can write the collocation system as

$$
\left[\mathcal{C}^{+} \Psi(\boldsymbol{x})-\operatorname{rdiag}(\boldsymbol{G}) \mathcal{C}^{-} \Psi(\boldsymbol{x})\right] \mathcal{F} \boldsymbol{U}=\operatorname{diag}(\boldsymbol{G})-I,
$$

where $\boldsymbol{G}=G(\boldsymbol{x})=\left(G\left(x_{1}\right), \ldots, G\left(x_{N}\right)\right)^{\top}$ and rdiag corresponds to matrix multiplication on the right:

$$
\operatorname{rdiag}\left(\begin{array}{c}
G_{1} \\
\vdots \\
G_{N}
\end{array}\right)\left(\begin{array}{c}
A_{1} \\
\vdots \\
A_{N}
\end{array}\right)=\left(\begin{array}{c}
A_{1} G_{1} \\
\vdots \\
A_{N} G_{N}
\end{array}\right)
$$

To be precise,

$$
\mathcal{C}^{ \pm} \Psi(\boldsymbol{x})=\left(\begin{array}{ccc}
\mathcal{C}^{ \pm} \psi_{1}\left(x_{1}\right) & \cdots & \mathcal{C}^{ \pm} \psi_{N}\left(x_{1}\right) \\
\vdots & \ddots & \vdots \\
\mathcal{C}^{ \pm} \psi_{1}\left(x_{N}\right) & \cdots & \mathcal{C}^{ \pm} \psi_{N}\left(x_{N}\right)
\end{array}\right)
$$

Note that the rows of $\boldsymbol{U}$ are typically row-vectors or matrices while $\mathcal{C}^{ \pm} \Psi(\boldsymbol{x})$ is a matrix whose entries are scalars. We treat these multiplications in the natural way: if $a_{i j}$ are scalars and $\boldsymbol{b}_{i}$ are row vectors or matrices, then

$$
\left(\begin{array}{ccc}
a_{11} & \cdots & a_{1 n} \\
\vdots & \ddots & \vdots \\
a_{n 1} & \cdots & a_{n n}
\end{array}\right)\left(\begin{array}{c}
\boldsymbol{b}_{1} \\
\vdots \\
\boldsymbol{b}_{n}
\end{array}\right)=\left(\begin{array}{c}
a_{11} \boldsymbol{b}_{1}+\cdots+a_{1 n} \boldsymbol{b}_{n} \\
\vdots \\
a_{n 1} \boldsymbol{b}_{1}+\cdots+a_{n n} \boldsymbol{b}_{n}
\end{array}\right)
$$

Once we have computed the values $\boldsymbol{U}$ by solving (2.2), we obtain an approximation to $\Phi$ :

$$
\Phi(z) \approx \tilde{\Phi}(z)=I+\mathcal{C} \Psi(z) \boldsymbol{U}
$$

The key piece in our framework is a method to compute the Cauchy transforms $\mathcal{C}^{ \pm} \Psi(\boldsymbol{x})$, where $\boldsymbol{x}$ and $\Psi$ are chosen appropriately. In [15] it was noted that the Cauchy transform can be computed numerically for $\Gamma=\mathbb{I}=[-1,1]$ uniformly throughout the complex plane, as well as the limits as $z$ approaches $\Gamma$, using the fast discrete cosine transform (DCT) and the Chebyshev basis. But this approach can be utilized for any curve conformally mappable to the unit interval. Furthermore,

$$
\mathcal{C}_{\Gamma_{1} \cup \Gamma_{2}}=\mathcal{C}_{\Gamma_{1}}+\mathcal{C}_{\Gamma_{2}}
$$

Thus we can also efficiently compute the Cauchy transform over any $\Gamma$ whose individual pieces are conformally mappable to the unit interval, such as all the curves in Figure 1. This 
motivates the choice of $\boldsymbol{x}$ and $\Psi$ as mapped Chebyshev points and polynomials, as discussed in Section 3.

In Section 4 we write down in closed form an expression for the Cauchy transform of Chebyshev polynomials, which allows the computation of the matrix $\mathcal{C}^{ \pm} \Psi(\boldsymbol{x})$. There is a critical snag: the points $\boldsymbol{x}$ contain junction points of the domain where the Cauchy transform can blow up, except under conditions on $\boldsymbol{U}$. Fortunately, we can resolve this issue in a manner that guarantees boundedness of $\mathcal{C}^{ \pm} \Psi(\boldsymbol{x}) \boldsymbol{U}$. It also means that the expression (2.2) is not the actual system we solve, but rather

$$
\left[C^{+}-\operatorname{rdiag}(\boldsymbol{G}) C^{-}\right] \boldsymbol{U}=\operatorname{diag}(\boldsymbol{G})-I
$$

for (scalar) matrices $C^{ \pm}$constructed in Section 5 .

Indeed, the presence of the junction points in $\boldsymbol{x}$ is crucial: it allows us to impose the conditions on $\boldsymbol{U}$ so that our approximation does not blow up. This - along with the use of exact formulæ for the Cauchy transform in place of quadrature - is the reason our approach avoids the difficulties seen in [6], where, by trying to avoid the junction point, exponential clustering near junction points was needed to simulate boundedness of the approximation.

In Section 6 we describe properties of the approximate system. The surprising fact is that (2.3) is sufficient to ensure boundedness of the solution, under a weak condition on the behaviour of the jump matrix $G$ at the junction points. In the rare case where this weak condition fails, (2.3) is automatically not of full rank, and we extend the linear system to enforce boundedness of the approximate solution.

In Section 7 we provide numerical experiments which demonstrate the effectiveness of the approach. We compute solutions to the Painlevé III and Painlevé IV ODEs, as the jump curves of the associated RH problems are sufficiently complicated - see Figure 1 to demonstrate the flexibility of the new framework.

A Mathematica implementation of the framework described in this paper is available online $[\mathbf{1 3}]$.

\section{Representation of jump matrices}

On the unit interval, we represent functions by their values at Chebyshev points of the second kind,

$$
\boldsymbol{x}^{\mathbb{I}}=\boldsymbol{x}^{\mathbb{I}, n}=\left(\begin{array}{c}
x_{1}^{\mathbb{I}, n} \\
\vdots \\
x_{n}^{\mathbb{I}, n}
\end{array}\right)=\left(\begin{array}{c}
-1 \\
\cos \pi\left(-1+\frac{1}{n-1}\right) \\
\vdots \\
\cos \pi\left(1-\frac{1}{n-1}\right) \\
1
\end{array}\right)
$$

As touched on before, by choosing points which include the endpoints, we have the ability to enforce that our approximation to $\Phi$ is bounded. 
The natural basis is now Chebshev polynomials of the first kind:

$$
\Psi^{\mathbb{I}}(z)=\left(T_{0}(z), \ldots, T_{n-1}(z)\right)
$$

The DCT, under appropriate scalings, is an $n \times n$ matrix $\mathcal{F}^{n}=\mathcal{F}$ such that

$$
\left(\begin{array}{c}
\check{U}_{0} \\
\vdots \\
\check{U}_{n-1}
\end{array}\right)=\mathcal{F} \boldsymbol{U}
$$

are the (row vector/matrix valued) Chebyshev coefficients of the polynomial which takes the values $\boldsymbol{U}$ at $\boldsymbol{x}^{\mathbb{I}}$ :

$$
\Psi^{\mathbb{I}}\left(\boldsymbol{x}^{\mathbb{I}}\right) \mathcal{F} \boldsymbol{U}=\sum_{k=0}^{n-1} \check{U}_{k} T_{k}\left(\boldsymbol{x}^{\mathbb{I}}\right)=\boldsymbol{U}
$$

In other words, $\mathcal{F}^{-1}=\Psi^{\mathbb{I}}\left(\boldsymbol{x}^{\mathbb{I}}\right)$ (though both $\mathcal{F}$ and its inverse can be applied in $\mathcal{O}(n \log n)$ time). The choice of Chebyshev polynomials is motivated by the fast transform and the explicit formulæ for their Cauchy transform described in the next section.

Now consider $\Gamma$ which can be conformally mapped to the unit interval, and let $M: \Gamma \rightarrow \mathbb{I}$ denote such a map. Example conformal maps are:

$$
\begin{aligned}
& \text { Interval }(a, b) \\
& M_{[a, b]}(z)=\frac{a+b-2 z}{a-b} \\
& \text { Stetched half line } \\
& M_{\left[a, \mathrm{e}^{\mathrm{i} \theta} \infty\right), L}(z)=\frac{a+\mathrm{e}^{\mathrm{i} \theta} L-z}{a-\mathrm{e}^{\mathrm{i} \theta} L-z} \\
& \text { Reverse stretched half line } \\
& M_{\left(\mathrm{e}^{\mathrm{i} \theta} \infty, a\right], L}(z)=-M_{\left[a, \mathrm{e}^{\mathrm{i} \theta} \infty\right), L}(z) \\
& \text { Arc } \\
& M_{a+r \mathrm{e}^{\mathrm{i}}\left[\theta_{1}, \theta_{2}\right]}(z)=\frac{\left(\mathrm{e}^{\frac{1}{2} \mathrm{i} \theta_{1}}+\mathrm{e}^{\frac{1}{2} \mathrm{i} \theta_{2}}\right)\left(\mathrm{e}^{\frac{1}{2} \mathrm{i}\left(\theta_{1}+\theta_{2}\right)} r-z+a\right)}{\left(\mathrm{e}^{\frac{1}{2} \mathrm{i} \theta_{1}}-\mathrm{e}^{\frac{1}{2} \mathrm{i} \theta_{2}}\right)\left(\mathrm{e}^{\frac{1}{2} \mathrm{i}\left(\theta_{1}+\theta_{2}\right)} r-z-a\right)} \\
& \text { Stretched interval } \\
& M_{[a, b], L}(z)=M_{[0, \infty), L}\left(M_{[0, \infty), 1}^{-1}\left(M_{[a, b]}(z)\right)\right) .
\end{aligned}
$$

Remark: In the examples below, we let the parameter $L=1$ in the stretched half line and will not use the stretched interval. However, in applying this approach to the RH problems from nonlinear steepest descent, this parameter is crucial to representing functions of the form $\mathrm{e}^{\omega g(z)}$ as $\omega$ becomes large.

If $\Gamma$ is bounded, (such as an interval or arc) then we can represent functions using the mapped Chebyshev basis

$$
\Psi^{\Gamma}(z)=\Psi^{\mathbb{I}}(M(z))=\left(T_{0}^{\Gamma}(z), \ldots, T_{n-1}^{\Gamma}(z)\right)=\left(T_{0}(M(z)), \ldots, T_{n-1}(M(z))\right)
$$


and the points $\boldsymbol{x}^{\Gamma}=M^{-1}\left(\boldsymbol{x}^{\mathbb{I}}\right)$. The transform from samples at $\boldsymbol{x}^{\Gamma}$ is simply $\mathcal{F}^{\Gamma}=\mathcal{F}$ again:

$$
\Psi^{\Gamma}\left(\boldsymbol{x}^{\Gamma}\right) \mathcal{F}^{\Gamma} \boldsymbol{U}=\Psi^{\mathbb{I}}(\boldsymbol{x}) \mathcal{F} \boldsymbol{U}=\boldsymbol{U} .
$$

However, when $\Gamma$ is unbounded (hence $M(\infty)$ is in $\mathbb{I}$, and for the conformal maps we consider always either -1 or +1 ) and $f$ decays at infinity, we will need expansion in a basis which captures this fact; otherwise, the Cauchy transform of the basis is not well-defined. This is straightforward:

$$
\begin{aligned}
f(z) & =\sum \check{f}_{k} T_{k}(M(z))=\sum \check{f}_{k} T_{k}(M(z))-\sum \check{f}_{k} T_{k}(M(\infty)) \quad(\text { since } f(\infty)=0) \\
& =\sum \check{f}_{k}\left[T_{k}(M(z))-T_{k}(M(\infty))\right]=\sum \check{f}_{k} T_{k}^{\Gamma}(z) .
\end{aligned}
$$

In other words, this alternative choice of basis does not change the expansion coefficients. Thus we define the (in this case $n+1$ column) basis as

$$
\Psi^{\Gamma}(z)=\Psi^{\mathbb{I}}(M(z))-\Psi^{\mathbb{I}}(M(\infty))=\left(T_{0}^{\Gamma}(z), \ldots, T_{n}^{\Gamma}(z)\right) .
$$

Since we assume the function decays at infinity, we need only the finite points: if $M(\infty)=$ -1 , then

$$
\boldsymbol{x}^{\Gamma}=M^{-1}\left(\boldsymbol{x}_{2: n+1}^{\mathbb{I}, n+1}\right)=M^{-1}\left(\left(x_{2}^{\mathbb{I}, n+1}, \ldots, x_{n+1}^{\mathbb{I}, n+1}\right)^{\top}\right)
$$

where we use the notation $i: j$ to denote the $i$ th through $j$ th row. The $(n+1) \times n$ transform operator $\mathcal{F}^{\Gamma}$ is $\mathcal{F}$ with its first column removed, or equivalently

$$
\mathcal{F}^{\Gamma} \boldsymbol{U}=\mathcal{F}_{1: n+1,2: n+1}^{n+1} \boldsymbol{U}=\mathcal{F}\left(\begin{array}{c}
0 \\
\boldsymbol{U}
\end{array}\right)
$$

where $i: j, k: l$ denotes the $i$ th through $j$ th row and $k$ th through $l$ th column. If $M(\infty)=+1$ then

$$
\boldsymbol{x}^{\Gamma}=M^{-1}\left(\boldsymbol{x}_{1: n}^{\mathbb{I}, n+1}\right)=M^{-1}\left(\left(x_{1}^{\mathbb{I}, n+1}, \ldots, x_{n}^{\mathbb{I}, n+1}\right)^{\top}\right)
$$

and

$$
\mathcal{F}^{\Gamma} \boldsymbol{U}=\mathcal{F}_{1: n+1,1: n}^{n+1} \boldsymbol{U}=\mathcal{F}\left(\begin{array}{c}
\boldsymbol{U} \\
0
\end{array}\right)
$$

What about functions which do not decay at infinity? For example, the original jump curve $G$ must approach the identity. Fortunately, we never need to compute the Cauchy transform of any curve which does not decay, nor its coefficients in a mapped Chebyshev basis; its values at the collocation points are sufficient.

The jump curve $\Gamma$ resulting from a $\mathrm{RH}$ problem is often a union of curves conformally mappable to the unit interval: for conformal maps $M_{\Gamma_{1}}, \ldots, M_{\Gamma_{\ell}}$, we have

$$
\Gamma=\Gamma_{1} \cup \cdots \cup \Gamma_{\ell}=M_{\Gamma_{1}}^{-1}(\mathbb{I}) \cup \cdots \cup M_{\Gamma_{\ell}}^{-1}(\mathbb{I}) .
$$


We can thus divide our solution vector as

$$
\boldsymbol{U}=\left(\begin{array}{c}
\boldsymbol{U}_{\Gamma_{1}} \\
\vdots \\
\boldsymbol{U}_{\Gamma_{\ell}}
\end{array}\right) \text { at the points } \boldsymbol{x}=\left(\begin{array}{c}
\boldsymbol{x}^{\Gamma_{1}} \\
\vdots \\
\boldsymbol{x}^{\Gamma_{\ell}}
\end{array}\right)
$$

We take the lengths of these vectors to be $n_{\Gamma_{1}}, \ldots, n_{\Gamma_{\ell}}$, so that $n_{\Gamma_{1}}+\cdots+n_{\Gamma_{\ell}}=N$, though in what follows the length is usually left implicit. We only permit the constituent curves of $\Gamma$ to overlap at their vertices; and these vertices are repeated in the vector $\boldsymbol{x}$. For brevity of notation, we will sometimes use the index as a sub/superscript, so that:

$$
M_{i}=M_{\Gamma_{i}}, \quad \boldsymbol{U}_{i}=\boldsymbol{U}_{\Gamma_{i}}, \quad \boldsymbol{x}^{i}=\boldsymbol{x}^{\Gamma_{i}} \quad \text { and } \quad n_{i}=n_{\Gamma_{i}} .
$$

We remark that in the typical $\mathrm{RH}$ problems from analysis, the jump curves $G$ are piecewise analytic, hence we will assume below that $G$ is analytic along each $\Gamma_{i}$. This condition can be relaxed significantly — indeed, for our computation of Cauchy transforms, uniform convergence of the Chebyshev polynomial is sufficient for bounded curves, along with weak conditions on asymptotic behaviour at infinity for unbounded curves, cf. [15] - however, in the examples considered, this condition is satisfied. Thus below we use sufficiently smooth to mean analytic along $\Gamma_{i}$, with a full asymptotic series (often all zero) at $\infty$ if $\Gamma_{i}$ is unbounded. Note that the columns of $\Psi^{\Gamma_{i}}$ are all sufficiently smooth.

\section{Cauchy transforms over intervals, rays and arcs}

Because we represent each of the constituent domains of $\Gamma$ by a conformal map to the unit interval, the initial goal is to compute the Cauchy transform for a function defined on the unit interval.

The Cauchy transform and Cauchy matrices over the unit interval

The Jacouwski map

$$
T(z)=\frac{1}{2}\left(z+\frac{1}{z}\right)
$$

maps the unit circle to the unit interval, with the interior and exterior of the circle both conformally mapped to the complex plane off the unit circle. We will need the following four inverses:

Map to the interior

Map to the exterior

Map to the lower half circle

Map to the upper half circle

$$
\begin{aligned}
& T_{+}^{-1}(x)=x-\sqrt{x-1} \sqrt{1+x}, \\
& T_{-}^{-1}(x)=x+\sqrt{x-1} \sqrt{1+x}, \\
& T_{\downarrow}^{-1}(x)=x-\mathrm{i} \sqrt{1-x} \sqrt{1+x}, \\
& T_{\uparrow}^{-1}(x)=x+\mathrm{i} \sqrt{1-x} \sqrt{1+x} .
\end{aligned}
$$

We know the exact formula for the Cauchy transform of the Chebyshev basis: 


\section{Theorem 4.1 Define}

$$
\begin{aligned}
\psi_{0}(z) & =\frac{2}{\mathrm{i} \pi} \operatorname{arctanh} z, \quad \mu_{k}(z)=\sum_{j=1}^{\left\lfloor\frac{k+1}{2}\right\rfloor} \frac{z^{2 j-1}}{2 j-1} \\
\psi_{k}(z) & =z^{k}\left[\psi_{0}(z)-\frac{2}{\mathrm{i} \pi}\left\{\begin{array}{cc}
\mu_{-k-1}(z) & \text { for } k<0 \\
\mu_{k}(1 / z) & \text { for } k>0
\end{array}\right]\right. \\
= & \frac{2}{\mathrm{i} \pi}\left\{\begin{array}{cc}
\frac{z^{1+2\lfloor-k / 2\rfloor+k}}{\left(1-z^{2}\right)(1+2\lfloor-k / 2\rfloor)} 2 F_{1}\left(\begin{array}{c}
1,1 \\
\frac{3}{2}+\lfloor-k / 2\rfloor
\end{array} ; \frac{z^{2}}{z^{2}-1}\right) & \text { for } k<0 \\
z^{k}\left(\operatorname{arctanh} z-\operatorname{arctanh} z^{-1}\right) & \text { for } k>0, \\
+\frac{z^{k-1-2\lfloor(k+1) / 2\rfloor}}{\left(1-z^{-2}\right)(1+2\lfloor(k+1) / 2\rfloor)}{ }_{2} F_{1}\left(\begin{array}{c}
1,1 \\
\frac{3}{2}+\lfloor(k+1) / 2\rfloor
\end{array} ; \frac{z^{-2}}{z^{-2}-1}\right) &
\end{array}\right.
\end{aligned}
$$

where ${ }_{2} F_{1}$ is the hypergeometric function [12]. Then

$$
\begin{aligned}
& \mathcal{C} T_{k}(x)=-\frac{1}{2}\left[\psi_{k}\left(T_{+}^{-1}(x)\right)+\psi_{-k}\left(T_{+}^{-1}(x)\right)\right], \\
& \mathcal{C} T_{k}(x) \underset{x \rightarrow-1}{\sim}-\frac{1}{2 \mathrm{i} \pi}(-1)^{k}[\log (-x-1)-\log 2]+\frac{1}{\mathrm{i} \pi}(-1)^{k}\left[\mu_{k-1}(-1)+\mu_{k}(-1)\right], \\
& \mathcal{C} T_{k}(x) \underset{x \rightarrow 1}{\sim} \frac{1}{2 \mathrm{i} \pi}[\log (x-1)-\log 2]+\frac{1}{\mathrm{i} \pi}\left[\mu_{k-1}(1)+\mu_{k}(1)\right],
\end{aligned}
$$

and, for $x \in \mathbb{I}$,

$$
\begin{aligned}
\mathcal{C}^{+} T_{k}(x) & =-\frac{1}{2}\left[\psi_{k}\left(T_{\downarrow}^{-1}(x)\right)+\psi_{-k}\left(T_{\downarrow}^{-1}(x)\right)\right] \\
& =-\frac{2}{\mathrm{i} \pi} T_{k}(x) \operatorname{arctanh} T_{\downarrow}^{-1}(x)+\frac{1}{\mathrm{i} \pi} \sum_{j=1}^{\left\lfloor\frac{k+1}{2}\right\rfloor} \frac{T_{k-2 j+1}(x)}{2 j-1} \begin{cases}1 & k-2 j+1=0 \\
2 & \text { otherwise }\end{cases} \\
\mathcal{C}^{-} T_{k}(x) & =-\frac{1}{2}\left[\psi_{k}\left(T_{\uparrow}^{-1}(x)\right)+\psi_{-k}\left(T_{\uparrow}^{-1}(x)\right)\right] \\
& =-\frac{2}{\mathrm{i} \pi} T_{k}(x) \operatorname{arctanh} T_{\uparrow}^{-1}(x)+\frac{1}{\mathrm{i} \pi} \sum_{j=1}^{\left\lfloor\frac{k+1}{2}\right\rfloor} \frac{T_{k-2 j+1}(x)}{2 j-1}\left\{\begin{array}{ll}
1 & k-2 j+1=0 \\
2 & \text { otherwise }
\end{array} .\right.
\end{aligned}
$$

Proof:

This theorem is a simplification of Theorem 6 from [15], which gave an expression for $\psi_{k}$ in terms of the Lerch transcendent function [12], found by summing the series definition of $\psi_{k}$. Here we use an equivalent formulation in terms of hypergeometric functions (since Mathematica can compute them more accurately). We also simplify the expression for $\mathcal{C} T_{k}$. Define

$$
\tilde{\psi}_{0}(z)=\frac{2}{\mathrm{i} \pi} \operatorname{arctanh} \frac{1}{z}
$$




$$
\tilde{\psi}_{k}(z)=z^{k}\left[\tilde{\psi}_{0}(z)-\frac{2}{\mathrm{i} \pi}\left\{\begin{array}{ll}
\mu_{-k-1}(z) & \text { for } k<0 \\
\mu_{k}(1 / z) & \text { for } k>0
\end{array}\right]\right.
$$

From [15] we have

$$
\mathcal{C} T_{k}(x)=-\frac{1}{4}\left[\psi_{k}\left(T_{+}^{-1}(x)\right)+\tilde{\psi}_{k}\left(T_{-}^{-1}(x)\right)+\psi_{-k}\left(T_{+}^{-1}(x)\right)+\tilde{\psi}_{-k}\left(T_{-}^{-1}(x)\right)\right] .
$$

However, $T_{-}^{-1}(x)=\frac{1}{T_{+}^{-1}(x)}$, therefore $\tilde{\psi}_{0}\left(T_{-}^{-1}(x)\right)=\psi_{0}\left(T_{+}^{-1}(x)\right)$. Furthermore, for $k>0$

$$
\begin{aligned}
\tilde{\psi}_{k}\left(T_{-}^{-1}(x)\right) & =T_{+}^{-1}(x)^{-k}\left[\psi_{0}\left(T_{+}^{-1}(x)\right)-\frac{2}{\mathrm{i} \pi} \mu_{k}\left(T_{+}^{-1}(x)\right)\right] \\
& =\psi_{-k}\left(T_{+}^{-1}(x)\right)+ \begin{cases}\frac{1}{2 k} & k \text { odd } \\
0 & k \text { even }\end{cases}
\end{aligned}
$$

and

$$
\tilde{\psi}_{-k}\left(T_{-}^{-1}(x)\right)=\psi_{k}\left(T_{+}^{-1}(x)\right)-\left\{\begin{array}{ll}
\frac{1}{2 k} & k \text { odd } \\
0 & k \text { even }
\end{array} .\right.
$$

It follows that

$$
\tilde{\psi}_{k}\left(T_{-}^{-1}(x)\right)+\tilde{\psi}_{-k}\left(T_{-}^{-1}(x)\right)=\psi_{k}\left(T_{+}^{-1}(x)\right)+\psi_{-k}\left(T_{+}^{-1}(x)\right) .
$$

The asymptotic behaviour at the endpoints was shown in [15]. The expression for $\mathcal{C}^{ \pm} T_{k}$ follows from the expression for $\mathcal{C} T_{k}$, the definition of $\mu_{k}$ and the fact that, for $z \in \mathbb{I}$, $\lim _{\epsilon \rightarrow 0+} T_{+}(z+\mathrm{i} \epsilon)=T_{\downarrow}^{-1}(x)$ and $\lim _{\epsilon \rightarrow 0+} T_{+}(z-\mathrm{i} \epsilon)=T_{\uparrow}^{-1}(x)$.

Q.E.D.

The Cauchy transform over curves other than the unit interval

On curves $\Gamma$ other than the unit interval, we use the conformal map $M=M_{\Gamma}$. The following theorem is trivially proved by considering the $\mathrm{RH}$ formulation of the Cauchy transform $\left(C^{+} f-C^{-} f=f\right.$ and $\left.C f(\infty)=0\right)$, cf. for example [15].

Theorem 4.2 Let $M: \Gamma \rightarrow \mathbb{I}$ be a conformal map, and let $g(x)=f(M(x))$. If $f$ is sufficiently smooth and either $\Gamma$ is bounded or $f(\infty)=0$, then

$$
\mathcal{C}_{\Gamma} f(z)=\mathcal{C}_{\mathbb{I}} g(M(z))-\mathcal{C}_{\mathbb{I}} g(M(\infty)) .
$$

In the case that $\Gamma$ is bounded, we immediately obtain

$$
\mathcal{C} T_{k}^{\Gamma}(z)=\mathcal{C} T_{k}(M(z))-\mathcal{C} T_{k}(M(\infty)) .
$$

When $\Gamma$ is unbounded we obtain (where $M(\infty)= \pm 1$ )

$$
\mathcal{C} T_{k}^{\Gamma}(z)=\mathcal{C}\left[T_{k}-T_{k}( \pm 1)\right](M(z))-\mathcal{C}\left[T_{k}-T_{k}( \pm 1)\right]( \pm 1)
$$




$$
=\mathcal{C} T_{k}(M(z))-( \pm 1)^{k} \mathcal{C} T_{0}(M(z))-\frac{( \pm 1)^{k}}{\mathrm{i} \pi}\left[\mu_{k}( \pm 1)+\mu_{k-1}( \pm 1)\right]
$$

Singularity data and the behaviour at endpoints

When we stitch the curve $\Gamma$ back together, we need to accurately know the behaviour of the Cauchy transform at the endpoints, where it typically blows up. We represent this using left and right singularity data

$$
\mathcal{S}^{\mathrm{L}} f, \mathcal{S}^{\mathrm{R}} f \in \mathbb{C}^{2} \times\{z \in \mathbb{C}:|z|=1\}
$$

We will use $\mathrm{L} / \mathrm{R}$ in conjunction with \pm , in which case $\mathrm{L}$ is identified with - and $\mathrm{R}$ is identified with + . If $z_{\mathrm{L} / \mathrm{R}}=M^{-1}( \pm 1)$ is the left/right endpoint of $\Gamma$ and $\{a, r, s\}=\mathcal{S}^{\mathrm{L} / \mathrm{R}} f$, then the Cauchy transform of $f$ behaves like

$$
\mathcal{C} f(z) \sim a+r \log s\left(z-z_{\mathrm{L} / \mathrm{R}}\right) \quad \text { as } \quad z \rightarrow z_{\mathrm{L} / \mathrm{R}} .
$$

From Theorem 4.1, we immediately see that

$$
\begin{aligned}
\mathcal{S}^{\mathrm{L}} T_{k} & =\left\{a_{k}^{\mathrm{L}}, r_{k}^{\mathrm{L}},-1\right\}=\left\{(-1)^{k} \frac{\log 2}{2 \mathrm{i} \pi}+\frac{(-1)^{k}}{\mathrm{i} \pi}\left[\mu_{k-1}(-1)+\mu_{k}(-1)\right],-\frac{(-1)^{k}}{2 \mathrm{i} \pi},-1\right\}, \\
\mathcal{S}^{\mathrm{R}} T_{k} & =\left\{a_{k}^{\mathrm{R}}, r_{k}^{\mathrm{R}}, 1\right\}=\left\{-\frac{\log 2}{2 \mathrm{i} \pi}+\frac{1}{\mathrm{i} \pi}\left[\mu_{k-1}(1)+\mu_{k}(1)\right], \frac{1}{2 \mathrm{i} \pi}, 1\right\} .
\end{aligned}
$$

For general $\Gamma$, we again use the conformal map. If $\Gamma$ is bounded, then, since $M(z) \sim$ $-1+M^{\prime}\left(z_{\mathrm{L}}\right)\left(z-z^{\mathrm{L}}\right)$, we have

$$
\begin{aligned}
\mathcal{C} T_{k}^{\Gamma}(z) & =\mathcal{C} T_{k}(M(z))-\mathcal{C} T_{k}(M(\infty)) \\
& \sim a_{k}^{\mathrm{L}}-\mathcal{C} T_{k}(M(\infty))+r_{k}^{\mathrm{L}} \log \left[-M^{\prime}\left(z_{\mathrm{L}}\right)\left(z-z_{\mathrm{L}}\right)\right] \\
& =a_{k}^{\mathrm{L}}-\mathcal{C} T_{k}(M(\infty))+r_{k}^{\mathrm{L}} \log \left|M^{\prime}\left(z_{\mathrm{L}}\right)\right|+r_{k}^{\mathrm{L}} \log \left[-\mathrm{e}^{\mathrm{i} \arg M^{\prime}\left(z_{\mathrm{L}}\right)}\left(z-z_{\mathrm{L}}\right)\right] .
\end{aligned}
$$

With a similar expression for the behaviour at $z^{\mathrm{R}}$, we find that

$$
\mathcal{S}^{\mathrm{L} / \mathrm{R}} T_{k}^{\Gamma}=\left\{a_{k}^{\mathrm{L} / \mathrm{R}}-\mathcal{C} T_{k}(M(\infty))+r_{k}^{\mathrm{L} / \mathrm{R}} \log \left|M^{\prime}\left(z_{\mathrm{L} / \mathrm{R}}\right)\right|, r_{k}^{\mathrm{L} / \mathrm{R}}, \pm \mathrm{e}^{\mathrm{i} \arg M^{\prime}\left(z_{\mathrm{L} / \mathrm{R}}\right)}\right\} .
$$

If $\Gamma$ is unbounded with $M(\infty)=1$, then, as $z \rightarrow z_{\mathrm{L}}$,

$$
\begin{gathered}
\mathcal{C} T_{k}^{\Gamma}(z)=\mathcal{C}\left[T_{k}-1\right](M(z))-\mathcal{C}\left[T_{k}-1\right](1) \\
\sim a_{k}^{\mathrm{L}}-a_{0}^{\mathrm{L}}-\frac{1}{\mathrm{i} \pi}\left[\mu_{k}(1)+\mu_{k-1}(1)\right]+r_{k}^{\mathrm{L}} \log \left[-M^{\prime}\left(z_{\mathrm{L}}\right)\left(z-z_{\mathrm{L}}\right)\right] \\
=a_{k}^{\mathrm{L}}-a_{0}^{\mathrm{L}}-\frac{1}{\mathrm{i} \pi}\left[\mu_{k}(1)+\mu_{k-1}(1)\right]+r_{k}^{\mathrm{L}} \log \left|M^{\prime}\left(z_{\mathrm{L}}\right)\right| \\
\quad+r_{k}^{\mathrm{L}} \log \left[-\mathrm{e}^{\mathrm{i} \arg M^{\prime}\left(z_{\mathrm{L}}\right)}\left(z-z_{\mathrm{L}}\right)\right] .
\end{gathered}
$$


Therefore

$$
\mathcal{S}^{\mathrm{L}} T_{k}^{\Gamma}=\left\{a_{k}^{\mathrm{L}}-a_{0}^{\mathrm{L}}-\frac{1}{\mathrm{i} \pi}\left[\mu_{k}(1)+\mu_{k-1}(1)\right]+r_{k}^{\mathrm{L}} \log \left|M^{\prime}\left(z_{\mathrm{L}}\right)\right|, r_{k}^{\mathrm{L}},-\mathrm{e}^{\mathrm{i} \arg M^{\prime}\left(z_{\mathrm{L}}\right)}\right\}
$$

and we leave the right singularity data (which corresponds to the behaviour at infinity) undefined. Likewise, if $M(\infty)=-1$ then

$$
\mathcal{S}^{\mathrm{R}} T_{k}^{\Gamma}=\left\{a_{k}^{\mathrm{R}}-(-)^{k} a_{0}^{\mathrm{R}}-\frac{(-)^{k}}{\mathrm{i} \pi}\left[\mu_{k}(-1)+\mu_{k-1}(-1)\right]+r_{k}^{\mathrm{R}} \log \left|M^{\prime}\left(z_{\mathrm{R}}\right)\right|, r_{k}^{\mathrm{R}}, \mathrm{e}^{\mathrm{i} \arg M^{\prime}\left(z_{\mathrm{R}}\right)}\right\}
$$

and the left singularity data is undefined.

We will use later that $r_{k}^{\mathrm{L} / \mathrm{R}}$ is independent of the curve $\Gamma$, and $s_{k}^{\mathrm{L} / \mathrm{R}}$ is always $-\mathrm{e}^{-\mathrm{i} \theta}$, where $\theta=-\arg \mp M^{\prime}\left(z_{\mathrm{L} / \mathrm{R}}\right)$ is the angle at which $\Gamma$ leaves $z_{\mathrm{L} / \mathrm{R}}$. Moreover, since $r_{k}^{\mathrm{L} / \mathrm{R}}=$ $\frac{( \pm 1)^{k+1}}{2 \mathrm{i} \pi}= \pm \frac{T_{k}( \pm 1)}{2 \mathrm{i} \pi}$ we have (here $m$ is the number of rows of $\mathcal{F}^{\Gamma}: m=n_{\Gamma}$ if $\Gamma$ is bounded, $m=n_{\Gamma}+1$ otherwise)

$$
\left(r_{0}^{\mathrm{L} / \mathrm{R}}, \ldots, r_{m-1}^{\mathrm{L} / \mathrm{R}}\right)^{\top} \mathcal{F}^{\Gamma} \boldsymbol{U}_{\Gamma}=\frac{ \pm}{2 \mathrm{i} \pi} \boldsymbol{e}_{\mathrm{L} / \mathrm{R}}^{\top} \boldsymbol{U}_{\Gamma}
$$

i.e., $\pm(2 \mathrm{i} \pi)^{-1}$ times the value at the corresponding endpoint. We use the notation $\boldsymbol{e}_{\mathrm{L}}=\boldsymbol{e}_{1}$ to denote the basis vector of length $n_{\Gamma}$ corresponding to $z_{\mathrm{L}}$ and $\boldsymbol{e}_{\mathrm{R}}=\boldsymbol{e}_{n_{\Gamma}}$ to denote the basis vector corresponding to $z_{\mathrm{R}}$.

For $z=z_{\mathrm{L} / \mathrm{R}}+p \mathrm{e}^{\mathrm{i} \theta}, p>0$ and $a, r, s=\mathcal{S}^{\mathrm{L} / \mathrm{R}} f$, we have, for $z \rightarrow z_{\mathrm{L} / \mathrm{R}}$,

$$
\mathcal{C} f(z) \sim a+r \log s p \mathrm{e}^{\mathrm{i} \theta}=a+\mathrm{i} r \arg s \mathrm{e}^{\mathrm{i} \theta}+r \log |p| .
$$

We define the finite part along angle $\theta$ as this expression with the logarithmic term thrown out:

$$
\mathrm{FP}_{\theta}^{\mathrm{L} / \mathrm{R}} f=a+\mathrm{i} r \arg s \mathrm{e}^{\mathrm{i} \theta} .
$$

As $\theta$ approaches $\arg s$, we have both a left and right limit. Thus we further define

$$
\mathrm{FP}_{ \pm}^{\mathrm{L}} f=a \mp r \mathrm{i} \pi \quad \text { and } \quad \mathrm{FP}_{ \pm}^{\mathrm{R}} f=a \pm r \mathrm{i} \pi
$$

\section{Constructing the Cauchy matrices}

We have established our representation of $U$ as $\boldsymbol{U}$, a vector of its values at the collocation points $\boldsymbol{x}$. To construct the collocation system, we have to evaluate the Cauchy transform of $\boldsymbol{U}$ again at the points $\boldsymbol{x}$. Thus we need to construct a matrix $C^{ \pm}$that we write as

$$
C^{ \pm} \boldsymbol{U}=\left(\begin{array}{ccc}
C^{ \pm}\left[\Gamma_{1}, \Gamma_{1}\right] & \cdots & C\left[\Gamma_{1}, \Gamma_{\ell}\right] \\
\vdots & \ddots & \vdots \\
C\left[\Gamma_{\ell}, \Gamma_{1}\right] & \cdots & C^{ \pm}\left[\Gamma_{\ell}, \Gamma_{\ell}\right]
\end{array}\right)\left(\begin{array}{c}
\boldsymbol{U}_{1} \\
\vdots \\
\boldsymbol{U}_{\ell}
\end{array}\right)
$$


For all $\Gamma$ :

$$
C^{-}[\Gamma, \Gamma] \quad C^{+}[\Gamma, \Gamma]-I
$$

For $\Gamma=\mathbb{I}$ :

$$
\begin{array}{ll}
C^{+}[\mathbb{I}, \mathbb{I}] & \text { Definition } 5.1 \\
C[\mathbb{I}, \Omega] \text { with } \Omega \text { disconnected from } \mathbb{I} & \text { Definition } 5.3 \\
C[\mathbb{I}, \Omega] \text { with } \Omega \text { connected to } \mathbb{I} & \text { Definition } 5.5
\end{array}
$$

For $\Gamma$ bounded with $M(\infty)=\infty$ :
$C^{+}[\Gamma, \Gamma]$
Definition 5.6
$C[\Gamma, \Omega]$ with $\Omega$ disconnected from $\Gamma$
Definition 5.7
$C[\Gamma, \Omega]$ with $\Omega$ connected to $\Gamma$
Definition 5.8

For $\Gamma$ bounded with $M(\infty) \neq \infty$ :
$C^{+}[\Gamma, \Gamma]$
Definition 5.9
$C[\Gamma, \Omega]$ with $\Omega$ disconnected from $\Gamma$
Definition 5.10
$C[\Gamma, \Omega]$ with $\Omega$ connected to $\Gamma$
Definition 5.11

For $\Gamma$ unbounded:
$C^{+}[\Gamma, \Gamma]$
Definition 5.12
$C[\Gamma, \Omega]$ with $\Omega$ disconnected from $\Gamma$
Definition 5.13
$C[\Gamma, \Omega]$ with $\Omega$ connected to $\Gamma$
Definition 5.14

Table 1: Definitions for the Cauchy matrices.

Since $\mathcal{C}^{+}-\mathcal{C}^{-}=I$, we require that $C^{+}-C^{-}=I$, or in particular we define $C^{-}\left[\Gamma_{i}, \Gamma_{i}\right]$ as $C^{+}\left[\Gamma_{i}, \Gamma_{i}\right]-I$. In other words, we only need to construct $C^{+}$.

We will sometimes simply refer to $C^{+}\left[\Gamma_{i}, \Gamma_{j}\right]$, which is $C^{+}\left[\Gamma_{i}, \Gamma_{j}\right]$ when $i=j$ and $C\left[\Gamma_{i}, \Gamma_{j}\right]$ otherwise. The goal of this section is to compute each $C^{+}\left[\Gamma_{i}, \Gamma_{j}\right]$ : an $n_{\Gamma_{j}} \times n_{\Gamma_{i}}$ matrix which maps from values $\boldsymbol{U}_{i}$ at the points $\boldsymbol{x}^{i}$ to something related to the Cauchy transform at the points $\boldsymbol{x}^{j}$.

In the remainder of this section, we speak in terms of general domains $\Gamma$ and $\Omega$. We will assume attached to each domain is a vector of points $\boldsymbol{x}^{\Gamma}$ and $\boldsymbol{x}^{\Omega}$, with lengths $n_{\Gamma}$ and $n_{\Omega}$. Because these lengths are not necessarily the same, the matrix $C[\Gamma, \Omega]$ is rectangular and of dimensions $n_{\Omega} \times n_{\Gamma}$. Note that we combine these rectangular matrices to construct the square matrix $C^{+}$.

We have several different situations to consider. See Table 1 for a guide for which definition is used to construct $C^{+}[\Gamma, \Omega]$. 
Our first concern is to construct $C^{+}[\mathbb{I}, \mathbb{I}]$, essentially the process of computing the limit of the Cauchy transform of a function defined on $\mathbb{I}$ back on to itself. Recall from Theorem 4.1 that

$$
\mathcal{C}^{+} T_{k}(x)=-\frac{2}{\mathrm{i} \pi} T_{k}(x) \operatorname{arctanh} T_{\downarrow}^{-1}(x)+\frac{1}{\mathrm{i} \pi} \sum_{j=1}^{\lfloor(k+1) / 2\rfloor} \frac{T_{k-2 j+1}(x)}{2 j-1} \begin{cases}1 & k-2 j+1=0 \\ 2 & \text { otherwise }\end{cases}
$$

The easiest term corresponds to the bounded term, which is a polynomial. For $n=n_{\mathbb{I}}$ odd, we can write it in terms of the following $n \times n$ almost Toeplitz matrix:

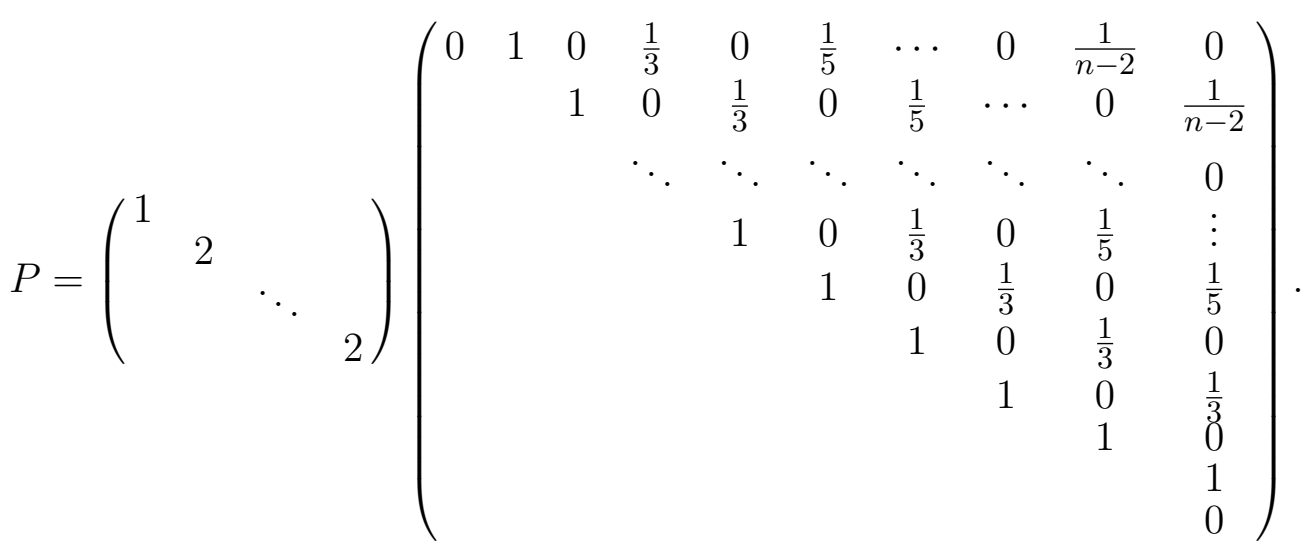

The version for $n$ odd is the Toeplitz matrix with its first row and column removed:

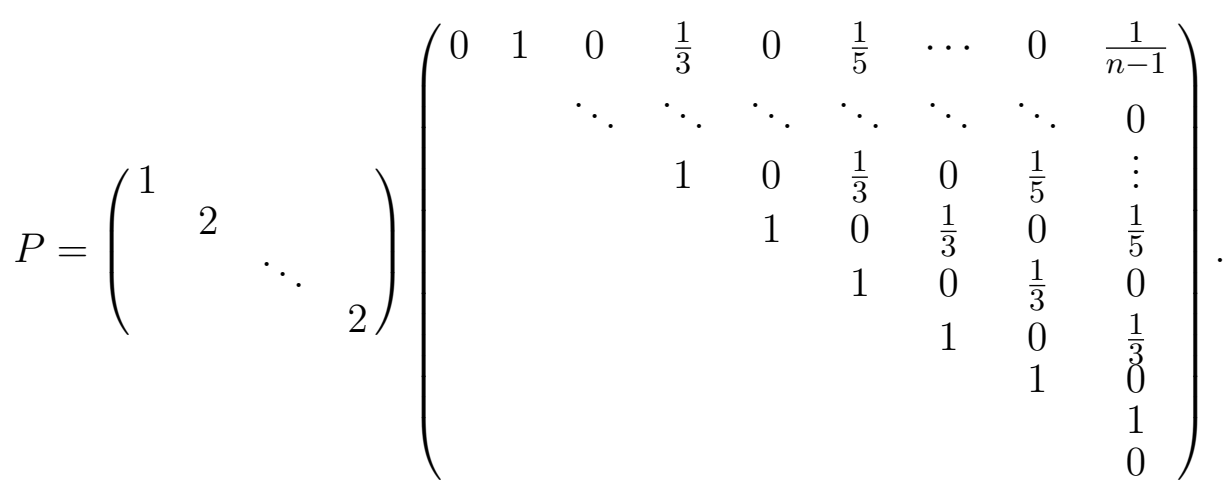

Now for the unbounded term, we note that we can simply evaluate the closed form expression for the Cauchy transform at $\boldsymbol{x}^{\mathbb{I}}$ with its endpoints removed: $\overline{\boldsymbol{x}}^{\mathbb{I}}=\boldsymbol{x}_{2: n-1}^{\mathbb{I}}$. All that remains is the value at the endpoints. We use the finite part of the singularity data for 
this. From the definition of singularity data and (4.4), we find that

$$
\begin{aligned}
\mathrm{FP}_{+}^{\mathrm{L}} \Psi^{\mathbb{I}}(z) \mathcal{F}= & \left(\mathrm{FP}_{+}^{\mathrm{L}} T_{0}, \ldots, \mathrm{FP}_{+}^{\mathrm{L}} T_{0}\right) \mathcal{F}=\left(a_{0}^{\mathrm{L}}-r_{0}^{\mathrm{L}} \mathrm{i} \pi, \ldots, a_{n-1}^{\mathrm{L}}-r_{n-1}^{\mathrm{L}} \mathrm{i} \pi\right) \mathcal{F} \\
= & -[\log 2+\mathrm{i} \pi]\left(r_{0}^{\mathrm{L}}, \ldots, r_{n-1}^{\mathrm{L}}\right) \mathcal{F} \\
& \quad+\frac{1}{\mathrm{i} \pi}\left(\mu_{0}(-1),-\mu_{0}(-1)-\mu_{1}(-1), \ldots,(-1)^{n-1}\left(\mu_{n-2}(-1)+\mu_{n-1}(-1)\right)\right) \mathcal{F} \\
= & \frac{1}{\mathrm{i} \pi}\left[\frac{\log 2}{2}+\frac{\mathrm{i} \pi}{2}\right] \boldsymbol{e}_{\mathrm{L}}^{\top}+\frac{1}{\mathrm{i} \pi} \boldsymbol{e}_{\mathrm{L}}^{\top} P \mathcal{F} \boldsymbol{U} .
\end{aligned}
$$

Likewise,

$$
\mathrm{FP}_{+}^{\mathrm{R}} \Psi^{\mathbb{I}}(z) \mathcal{F}=\frac{1}{\mathrm{i} \pi}\left[-\frac{\log 2}{2}+\frac{\mathrm{i} \pi}{2}\right] \boldsymbol{e}_{\mathrm{R}}^{\top}+\frac{1}{\mathrm{i} \pi} \boldsymbol{e}_{\mathrm{R}}^{\top} P \mathcal{F} \boldsymbol{U} .
$$

We thus obtain

\section{Definition 5.1}

$$
C^{+}[\mathbb{I}, \mathbb{I}]=\frac{1}{\mathrm{i} \pi}\left(\begin{array}{lll}
\frac{\log 2}{2}+\frac{\mathrm{i} \pi}{2} & \\
& -2 \operatorname{diag}\left(\operatorname{arctanh} T_{\downarrow}^{-1}\left(\overline{\boldsymbol{x}}^{\mathbb{I}}\right)\right) & \\
& -\frac{\log 2}{2}+\frac{\mathrm{i} \pi}{2}
\end{array}\right)+\frac{1}{\mathrm{i} \pi} \mathcal{F}^{-1} P \mathcal{F}
$$

Remark: If instead of constructing a matrix, we were concerned with evaluating the limit of the Cauchy transform from the left or right (which together give the finite Hilbert transform), an important observation that was missed in [15] is that $P$ is a Toeplitz matrix plus a vector that only depends on the first row. In other words, we can evaluate the finite Hilbert transform of a function defined at the $n$ Chebyshev points $\boldsymbol{x}^{\mathbb{I}}$ at the same points in $\mathcal{O}(n \log n)$ time.

We now consider the Cauchy matrix $C[\mathbb{I}, \Gamma]$, for $\Gamma$ not connected to $\mathbb{I}$. If $n=n_{\mathbb{I}}$ is the degree of the Chebyshev interpolation, we require $\psi_{1-n}\left(T_{+}^{-1}\left(\boldsymbol{x}^{\Gamma}\right)\right), \ldots, \psi_{n-1}\left(T_{+}^{-1}\left(\boldsymbol{x}^{\Gamma}\right)\right)$. Since computing hypergeometric functions is slow, we want to avoid their computation as much as possible. Fortunately from the first definition of $\psi_{k}$, we have a simple recurrence relationship:

$$
\psi_{k}(z)=z \psi_{k-1}(z)-\left\{\begin{array}{ll}
\frac{2}{\mathrm{i} \pi k} & \text { for odd } k \\
0 & \text { otherwise }
\end{array} .\right.
$$

For $k>0$, we can first compute $\psi_{0}(z)$ via the arctanh function, and can in turn compute $\psi_{1}(z), \psi_{2}(z), \ldots$. For $k<0$, we cannot start with the arctanh function and use the recurrence relationship in reverse: each term is subtracting out the Taylor series of the arctanh function, hence round-off error occurs. Instead, we compute $\psi_{1-n}(z)$ using the hypergeometric function representation, followed by the recurrence relationship again in the forward direction. This leads to the following algorithm: 
Algorithm 5.2 Recurrence relationship

Given $\boldsymbol{z} \in \mathbb{C}^{m}$ and $n$, compute the $m \times n$ matrix $\boldsymbol{\Psi}_{n}^{z}$ as follows:

$$
\begin{aligned}
\boldsymbol{\psi}_{1-n} & =\psi_{1-n}(\boldsymbol{z}), \\
\boldsymbol{\psi}_{k} & =\boldsymbol{z} \boldsymbol{\psi}_{k-1}-\left\{\begin{array}{ll}
\frac{2}{\mathrm{i} \pi k} & \text { for odd } k \\
0 & \text { otherwise }
\end{array} \text { for } k=2-n, \ldots,-1,\right. \\
\boldsymbol{\psi}_{0} & =\psi_{0}(\boldsymbol{z}), \\
\boldsymbol{\psi}_{k} & =\boldsymbol{z} \boldsymbol{\psi}_{k-1}-\left\{\begin{array}{ll}
\frac{2}{\mathrm{i} \pi k} & \text { for odd } k \\
0 & \text { otherwise }
\end{array} \text { for } k=1, \ldots, n-1,\right. \\
\boldsymbol{\Psi}_{n}^{\boldsymbol{z}} & =-\frac{1}{2}\left(2 \boldsymbol{\psi}_{0}, \boldsymbol{\psi}_{-1}+\boldsymbol{\psi}_{1}, \ldots, \boldsymbol{\psi}_{1-n}+\boldsymbol{\psi}_{n-1}\right) .
\end{aligned}
$$

We can now successfully construct the necessary matrix:

Definition 5.3 If $\Gamma$ is not connected to $\mathbb{I}$, then, for $\boldsymbol{z}=T_{+}^{-1}\left(\boldsymbol{x}^{\Gamma}\right)$ and $n=n_{\mathbb{I}}$, define

$$
C[\mathbb{I}, \Gamma]=\Psi_{n}^{z} \mathcal{F}
$$

Now suppose the curve $\Gamma$ is connected to the unit interval at one of its endpoints, for example the left endpoint of $\Gamma$ is either $\pm 1: M_{\Gamma}( \pm 1)=-1$. We can determine $\Psi_{n}^{z}$, but now with all points in $\boldsymbol{x}^{\Gamma}$ not connected to $\mathbb{I}: T_{+}^{-1}\left(\boldsymbol{x}_{2: n_{\Gamma}}^{\Gamma}\right)=T_{+}^{-1}\left(\left(x_{2}^{\Gamma}, \ldots, x_{n_{\Gamma}}^{\Gamma}\right)^{\top}\right)$. Then we take the finite part of the remaining term, using the fact that the angle that $\Gamma$ leaves \pm 1 is $-\arg M_{\Gamma}^{\prime}( \pm 1)$. To compute this, we will need the values

$$
\mu_{0}( \pm 1), \pm\left[\mu_{1}( \pm 1)+\mu_{0}( \pm 1)\right], \ldots,( \pm)^{n-1}\left[\mu_{1}( \pm 1)+\mu_{0}( \pm 1)\right]
$$

These are evaluated by simply summing the series term by term:

Algorithm 5.4 Taylor series

Given $n$, compute $\boldsymbol{\mu}_{n}^{\mathrm{L} / \mathrm{R}}$ (again, relating $\mathrm{L}$ with -1 and $\mathrm{R}$ with +1 ) as follows:

$$
\begin{aligned}
\mu_{0} & =0 \\
\mu_{k} & =\mu_{k-1}+\left\{\begin{array}{ll}
\frac{( \pm 1)^{k}}{k} & \text { for odd } k \\
0 & \text { otherwise }
\end{array} \text { for } \quad k=1, \ldots, n-1\right. \\
\boldsymbol{\mu}_{n}^{\mathrm{L} / \mathrm{R}} & =\frac{1}{\mathrm{i} \pi}\left(\mu_{0}, \pm\left[\mu_{0}+\mu_{1}\right], \ldots( \pm 1)^{n-1}\left[\mu_{n-2}+\mu_{n-1}\right]\right) .
\end{aligned}
$$


Definition 5.5 If $M_{\Gamma}( \pm 1)=-1$, then, for $\theta=-\arg M_{\Gamma}^{\prime}( \pm 1), \boldsymbol{z}=T_{+}^{-1}\left(\boldsymbol{x}_{2: n_{\Gamma}}^{\Gamma}\right), n=n_{\mathbb{I}}$ and

$$
\boldsymbol{r}^{\mathrm{L} / \mathrm{R}}=\left(r_{0}^{\mathrm{L} / \mathrm{R}}, \ldots, r_{n-1}^{\mathrm{L} / \mathrm{R}}\right)=\left(\frac{ \pm 1}{2 \mathrm{i} \pi}, \ldots, \frac{( \pm 1)^{n-1}}{2 \mathrm{i} \pi}\right)
$$

define

$$
C[\mathbb{I}, \Gamma]=\left(\frac{\boldsymbol{\mu}_{n}^{\mathrm{L} / \mathrm{R}}+\left[\mathrm{i} \arg \left( \pm \mathrm{e}^{\mathrm{i} \theta}\right)-\log 2\right] \boldsymbol{r}^{\mathrm{L} / \mathrm{R}}}{\boldsymbol{\Psi}_{n}^{z}}\right) \mathcal{F}
$$

If $M_{\Gamma}( \pm 1)=1$, then, for $\theta=-\arg \left(-M_{\Gamma}^{\prime}( \pm 1)\right), \boldsymbol{z}=T_{+}^{-1}\left(\boldsymbol{x}_{1: n_{\Gamma}-1}\right)$ and $n=n_{\mathbb{I}}$, define

$$
C[\mathbb{I}, \Gamma]=\left(\begin{array}{c}
\Psi_{n}^{z} \\
\boldsymbol{\mu}_{n}^{\mathrm{L} / \mathrm{R}}+\left[\mathrm{i} \arg \left( \pm \mathrm{e}^{\mathrm{i} \theta}\right)-\log 2\right] \boldsymbol{r}^{\mathrm{L} / \mathrm{R}}
\end{array}\right) \mathcal{F}
$$

The construction in the case both endpoints are connected to $\mathbb{I}$ is also clear, though omitted.

Curves $\Gamma$ whose conformal map leaves $\infty$ at $\infty$

We now consider the matrices $C^{+}[\Gamma, \Omega]$ such that $M_{\Gamma}(\infty)=\infty$. This naturally precludes the case where $\Gamma$ is unbounded. From Theorem 4.2, we know that the Cauchy matrix is essentially unchanged, except for alterations of the singularity data. Thus we obtain the following constructions:

Definition 5.6 If $M_{\Gamma}(\infty)=\infty$, then, for $z_{\mathrm{L}}=M_{\Gamma}^{-1}(-1)$ and $z_{\mathrm{R}}=M_{\Gamma}^{-1}(+1)$, define

$$
C^{+}[\Gamma, \Gamma]=C^{+}[\mathbb{I}, \mathbb{I}]+\frac{1}{2 \mathrm{i} \pi}\left(\begin{array}{ll}
-\log \left|M_{\Gamma}^{\prime}\left(z_{\mathrm{L}}\right)\right| & \\
& \log \left|M_{\Gamma}^{\prime}\left(z_{\mathrm{R}}\right)\right|
\end{array}\right) .
$$

Definition 5.7 If $M_{\Gamma}(\infty)=\infty$ and $\Gamma$ and $\Omega$ are disconnected, then define

$$
C[\Gamma, \Omega]=C\left[\mathbb{I}, M_{\Gamma}(\Omega)\right]
$$

where $M_{\Gamma}(\Omega)$ is the domain resulting from conformally mapping $\Omega$ using $M_{\Gamma}$.

Definition 5.8 Suppose $M_{\Gamma}(\infty)=\infty$, and let $z_{\mathrm{L}}=M_{\Gamma}^{-1}(-1)$ and $z_{\mathrm{R}}=M_{\Gamma}^{-1}(+1)$. If $z_{\mathrm{L}}=M_{\Omega}^{-1}(-1)$, then define

$$
C[\Gamma, \Omega]=C\left[\mathbb{I}, M_{\Gamma}(\Omega)\right]-\frac{\log \left|M_{\Gamma}^{\prime}\left(z_{\mathrm{L}}\right)\right|}{2 \mathrm{i} \pi}(1)
$$


where the last matrix is the $n_{\Omega} \times n_{\Gamma}$ matrix with a one in the $(1,1)$ entry and zeros elsewhere (similar notation is used below). If $z_{\mathrm{L}}=M_{\Omega}^{-1}(+1)$, then define

$$
C[\Gamma, \Omega]=C[\mathbb{I}, M(\Omega)]-\frac{\log \left|M_{\Gamma}^{\prime}\left(z_{\mathrm{L}}\right)\right|}{2 \mathrm{i} \pi}\left(\begin{array}{l} 
\\
1
\end{array}\right) .
$$

If $z_{\mathrm{R}}=M_{\Omega}^{-1}(-1)$, then define

$$
C[\Gamma, \Omega]=C\left[\mathbb{I}, M_{\Gamma}(\Omega)\right]+\frac{\log \left|M_{\Gamma}^{\prime}\left(z_{\mathrm{R}}\right)\right|}{2 \mathrm{i} \pi}\left(\begin{array}{l}
1 \\
\end{array}\right)
$$

If $z_{\mathrm{R}}=M_{\Omega}^{-1}(+1)$, then define

$$
C[\Gamma, \Omega]=C\left[\mathbb{I}, M_{\Gamma}(\Omega)\right]+\frac{\log \left|M_{\Gamma}^{\prime}\left(z_{\mathrm{R}}\right)\right|}{2 \mathrm{i} \pi}\left(\begin{array}{l} 
\\
\quad 1
\end{array}\right)
$$

\section{Bounded curves}

In this section, we assume that $M_{\Gamma}(\infty) \neq \infty$, but where $\Gamma$ is bounded. From (4.1), we know we must subtract out the behaviour of the Cauchy transform of where $\infty$ is mapped to under $M_{\Gamma}$, and alter the finite part at the endpoints.

Definition 5.9 For $z=T_{+}^{-1}\left(M_{\Gamma}(\infty)\right)$ and $n=n_{\Gamma}$, define

$$
C^{+}[\Gamma, \Gamma]=\{\text { Definition } 5.6\}-\mathbf{1}_{n \times n} \operatorname{diag}\left(\mathbf{\Psi}_{n}^{z}\right) \mathcal{F},
$$

where $\mathbf{1}_{k \times l}$ is the $k \times l$ matrix of all ones, and in this case $\boldsymbol{\Psi}_{n}^{z}$ is a simply a row vector.

Definition 5.10 If $\Gamma$ and $\Omega$ are disconnected, then, for $z=T_{+}^{-1}\left(M_{\Gamma}(\infty)\right)$ and $n=n_{\Gamma}$, define

$$
C[\Gamma, \Omega]=C\left[\mathbb{I}, M_{\Gamma}(\Omega)\right]-\mathbf{1}_{n_{\Omega} \times n} \operatorname{diag}\left(\boldsymbol{\Psi}_{n}^{z}\right) \mathcal{F}
$$

Definition 5.11 If $\Gamma$ and $\Omega$ are connected,then, for $z=T_{+}^{-1}\left(M_{\Gamma}(\infty)\right)$ and $n=n_{\Gamma}$, define

$$
C[\Gamma, \Omega]=\{\text { Definition } 5.8\}-\mathbf{1}_{n_{\Omega} \times n} \operatorname{diag}\left(\boldsymbol{\Psi}_{n}^{z}\right) \mathcal{F} \text {. }
$$

\section{Unbounded curves}

We now consider the case where $\Gamma$ is unbounded. In this case $M(\infty)$ is \pm 1 , hence Algorithm 5.2 cannot be used, since the arctanh function blows up. But we know the basis vanishes at $\infty$, thus we simply take the finite part. 
Definition 5.12 If $M_{\Gamma}(\infty)=-1$, then, for $z_{\mathrm{R}}=M_{\Gamma}^{-1}(+1), n=n_{\Gamma}$ and $m=n_{\mathbb{I}}=n+1$, define

$$
C^{+}[\Gamma, \Gamma]=C^{+}[\mathbb{I}, \mathbb{I}]_{2: m, 2: m}-\mathbf{1}_{n \times m} \operatorname{diag}\left(\boldsymbol{\mu}_{m}^{\mathrm{L}}\right) \mathcal{F}^{\Gamma}+\frac{\log \left|M_{\Gamma}^{\prime}\left(z_{\mathrm{R}}\right)\right|}{2 \mathrm{i} \pi}\left(\begin{array}{l} 
\\
\quad 1
\end{array}\right) .
$$

If $M_{\Gamma}(\infty)=+1$, then, for $z_{\mathrm{L}}=M_{\Gamma}^{-1}(-1), n=n_{\Gamma}$ and $m=n_{\mathbb{I}}=n+1$, define

$$
C^{+}[\Gamma, \Gamma]=C^{+}[\mathbb{I}, \mathbb{I}]_{1: n, 1: n}-\mathbf{1}_{n \times m} \operatorname{diag}\left(\boldsymbol{\mu}_{m}^{\mathrm{R}}\right) \mathcal{F}^{\Gamma}-\frac{\log \left|M_{\Gamma}^{\prime}\left(z_{\mathrm{L}}\right)\right|}{2 \mathrm{i} \pi}(1) .
$$

Definition 5.13 Suppose $\Gamma$ and $\Omega$ are disconnected and let $n=n_{\Gamma}$ and $m=n_{\mathbb{I}}=n+1$. If $M_{\Gamma}(\infty)=-1$, then define

$$
C[\Gamma, \Omega]=C\left[\mathbb{I}, M_{\Gamma}(\Omega)\right]_{1: n_{\Omega}, 2: m}-\mathbf{1}_{n_{\Omega} \times m} \operatorname{diag}\left(\boldsymbol{\mu}_{m}^{\mathrm{L}}\right) \mathcal{F}^{\Gamma} .
$$

If $M_{\Gamma}(\infty)=+1$, then define

$$
C[\Gamma, \Omega]=C\left[\mathbb{I}, M_{\Gamma}(\Omega)\right]_{1: n_{\Omega}, 1: n}-\mathbf{1}_{n_{\Omega} \times m} \operatorname{diag}\left(\boldsymbol{\mu}_{m}^{\mathrm{R}}\right) \mathcal{F}^{\Gamma}
$$

Definition 5.14 Suppose $\Gamma$ and $\Omega$ are disconnected and let $n=n_{\Gamma}$ and $m=n_{\mathbb{I}}=n+1$. If $M_{\Gamma}(\infty)=-1$, then define

$$
C[\Gamma, \Omega]=\{\text { Definition } 5.8\}_{1: n_{\Omega}, 2: m}-\mathbf{1}_{n_{\Omega} \times m} \operatorname{diag}\left(\boldsymbol{\mu}_{m}^{\mathrm{L}}\right) \mathcal{F}^{\Gamma} .
$$

If $M_{\Gamma}(\infty)=+1$, then define

$$
C[\Gamma, \Omega]=\{\text { Definition } 5.8\}_{1: n_{\Omega}, 1: n}-\mathbf{1}_{n_{\Omega} \times m} \operatorname{diag}\left(\boldsymbol{\mu}_{m}^{\mathrm{R}}\right) \mathcal{F}^{\Gamma} .
$$

\section{Properties of the approximate solution}

We have described how we construct the matrices $C^{ \pm}$, thus we have everything necessary to construct the collocation system (2.3). Assuming it is nonsingular - which is satisfied, at least, for $G$ sufficiently close to the identity matrix - we can solve this system to obtain $\boldsymbol{U}$ using a dense linear algebra package (in our case, we use MATHEMATICA's built-in LinearSolve, which is based on LAPACK). We thus obtain

$$
\tilde{\Phi}(z)=I+\mathcal{C} \Phi(z) \mathcal{F} \boldsymbol{U}=I+\sum_{k=1}^{\ell} \mathcal{C} \Phi^{\Gamma_{k}}(z) \mathcal{F}^{\Gamma_{k}} \boldsymbol{U}_{k},
$$

and each of these Cauchy transforms is computable, as described in Section 4. 
We must justify why $\tilde{\Phi}$ can be considered an approximation to $\Phi$. One posible issue is that $\tilde{\Phi}$ is, as far as we know, unbounded. Another issue is that the values we assign for $C^{ \pm}$at the junction points of $\Gamma$ are, a priori, unrelated to the value of the Cauchy transform of our basis, which blows up. The key to justifying the approximation is the following condition, which, when satisfied, will resolve both issues:

Definition 6.1 Given $\xi \in \mathbb{C}$, let $\left\{\Omega_{1}, \ldots, \Omega_{L}\right\}$ be the subset of $\left\{\Gamma_{1}, \ldots, \Gamma_{\ell}\right\}$ whose elements contain $\xi$ as an endpoint. Define $p_{i}=M_{\Omega_{i}}(\xi)= \pm 1$, which determines if it is the left or right endpoint, and $U_{i}=\boldsymbol{e}_{p_{i}}^{\top} \boldsymbol{U}_{\Omega_{i}}$, which is the value of the computed jump curve at $\xi$ (using the convention that $\left.\boldsymbol{e}_{-1}=\boldsymbol{e}_{n_{i}}\right)$. The zero sum condition at $\xi$ is satisfied if

$$
\sum_{i=1}^{L} p_{i} U_{i}=0
$$

The zero sum condition is satisfied if the zero sum condition at every endpoint of $\Gamma_{1}, \ldots, \Gamma_{\ell}$ is satisfied.

Lemma 6.2 Suppose that the zero sum condition is satisfied. Then $\tilde{\Phi}(z)=I+\mathcal{C} \Psi(z) \mathcal{F} \boldsymbol{U}$ is bounded and

$$
C^{ \pm} \boldsymbol{U}=\left(\begin{array}{c}
\lim _{\boldsymbol{z} \rightarrow \boldsymbol{x}^{1}} \text { for } \boldsymbol{z} \text { on } \Gamma_{1} I+\mathcal{C}^{ \pm} \Psi(\boldsymbol{z}) \mathcal{F} \boldsymbol{U} \\
\vdots \\
\lim _{\boldsymbol{z} \rightarrow \boldsymbol{x}^{\ell}} \text { for } \boldsymbol{z} \text { on } \Gamma_{\ell} I+\mathcal{C}^{ \pm} \Psi(\boldsymbol{z}) \mathcal{F} \boldsymbol{U}
\end{array}\right)
$$

i.e. applying $C^{ \pm}$is equivalent to computing the limit of the Cauchy transform of our approximation at the points $\boldsymbol{x}$.

Proof:

The only possible blow-up of the Cauchy transform is at an endpoint $\xi$ of the curves which make up $\Gamma$. We will use the notation of Definition 6.1, and let $\theta_{i}=-\arg \left[-p_{i} M_{\Omega_{i}}^{\prime}(\xi)\right]$ be the angle at which $\Omega_{i}$ leaves $\xi$. We have

$$
\tilde{\Phi}(z)=I+\mathcal{C} \Psi(z) \mathcal{F} \boldsymbol{U}=I+\sum_{k=1}^{\ell} \mathcal{C} \Psi^{k}(z) \mathcal{F}^{k} \boldsymbol{U}_{k} .
$$

As $z$ approaches $\xi$, we note that the Cauchy transform of any curve not connected to $\xi$ must be bounded at $\xi$, hence

$$
\lim _{\epsilon \rightarrow 0} \tilde{\Phi}(\xi+\epsilon)=D+\lim _{\epsilon \rightarrow 0} \sum_{i=1}^{L} \mathcal{C} \Psi^{\Omega_{i}}(\xi+\epsilon) \mathcal{F}^{\Omega_{i}} U_{\Omega_{i}} .
$$


Now each of these blows up at $\xi$, however, we know precisely the singularity data. Thus, for constants $D^{(i)}$, depending only, possibly, on $\arg \epsilon$, we have

$$
\begin{aligned}
\mathcal{C} \Psi^{\Omega_{i}}(\xi+\epsilon) \mathcal{F}^{\Omega_{i}} \boldsymbol{U}_{\Omega_{i}} & \sim D^{(1)}+\log \left(p_{i} \mathrm{e}^{-\mathrm{i} \theta_{i}} \epsilon\right)\left(r_{0}^{p_{i}}, \ldots, r_{n_{\Omega_{i}}-1}^{p_{i}}\right)^{\top} \mathcal{F}^{\Omega_{i}} \boldsymbol{U}_{\Omega_{i}} \\
& =D^{(2)}+\left[\log |\epsilon|+\mathrm{i} \arg \left(p_{i} \mathrm{e}^{-\mathrm{i} \theta_{i}} \epsilon\right)\right] \frac{p_{i} U_{i}}{2 \pi \mathrm{i}} \quad(\text { due to }(4.4)) \\
& =D_{\arg \epsilon}^{(3)}+\log |\epsilon| \frac{p_{i} U_{i}}{2 \pi \mathrm{i}}
\end{aligned}
$$

Thus we have

$$
\tilde{\Phi}(\xi+\epsilon) \sim D_{\arg \epsilon}^{(4)}+\frac{\log |\epsilon|}{2 \pi \mathrm{i}} \sum_{i=1}^{L} p_{i} U_{i}=D_{\arg \epsilon}^{(4)}
$$

which proves that it is bounded at $\xi$.

The value we assigned to the Cauchy matrices $C^{ \pm}\left[\Gamma_{i}, \Gamma_{j}\right]$ at points which are not junction points was, by definition, the Cauchy transform. The value we chose at each endpoint was chosen to be the limit of the Cauchy transform, ignoring the unbounded term which grew like $\log |z|$. We have shown that each of these unbounded terms is cancelled, therefore, the second part of the lemma follows.

Q.E.D.

What is clear from this lemma is that it is important that the zero sum condition is satisfied in order for our approximation to $\Phi$ to be analytic, hence a reasonable approximation to the actual $\Phi$. Now we have not (as of yet) imposed the zero sum condition; however, the following condition on the jump curve $G$ (hence completely independent of the discretization), which should almost always be satisfies, will automatically imply that the zero sum condition is satisfied:

Definition 6.3 Let $\left\{\Omega_{1}, \ldots, \Omega_{L}\right\}$ denote the subset of $\left\{\Gamma_{1}, \ldots, \Gamma_{\ell}\right\}$ whose elements contain $\xi$ as an endpoint, $\theta_{i}$ the angle at which $\Omega_{k}$ leaves $\xi, p_{i}=+1$ if $\xi$ is the left endpoint and -1 if $\xi$ is the right endpoint of $\Omega_{i}$ and let $G_{i}$ be the limit of $G(z)$ as $z$ approaches $\xi$ along $\Omega_{i}$. We assume that $\left\{\Omega_{1}, \ldots, \Omega_{L}\right\}$ is sorted so that $\theta_{i}$ is strictly increasing. The nonsingular junction condition at $\xi$ is satisfied if

$$
\left(\theta_{L}-\theta_{1}\right) I+\sum_{k=2}^{L}\left(\theta_{k-1}-\theta_{k}\right) G_{k}^{p_{k}} \cdots G_{L}^{p_{L}}
$$

is nonsingular. The nonsingular junction condition is satisfied if the nonsingular junction condition at every endpoint of $\Gamma_{1}, \ldots, \Gamma_{\ell}$ is satisfied. 
Theorem 6.4 Suppose that the $R H$ problem is well-posed, the collocation system has a solution and the nonsingular junction condition is satisfied. Then the computed vector $\boldsymbol{U}$ satisfies the zero sum condition and Lemma 6.2 applies.

Proof:

This theorem is a generalization of Theorem 4.2 from [14]. We reuse the notation of Lemma 6.2. For simplicity in notation, we assume every curve $\Omega_{1}, \ldots, \Omega_{L}$ has $\xi$ as a left endpoint; the generalization for other cases is straightforward.

Note that (since $\theta_{i}<\theta_{j}$ for $i<j$ ) the finite part of each $\boldsymbol{U}_{\Omega_{i}}$ along $\Omega_{j}$ is

$$
\mathrm{FP}_{\theta_{j}}^{\mathrm{L} / \mathrm{R}} \boldsymbol{U}_{\Omega_{i}}=a_{i}+\mathrm{i} r_{i} \arg \left(-\mathrm{e}^{\mathrm{i}\left(\theta_{j}-\theta_{i}\right)}\right)=a_{i}+\mathrm{i} r_{i}\left\{\begin{array}{ll}
\theta_{j}-\theta_{i}-\pi & \text { if } i<j \\
\theta_{j}-\theta_{i}+\pi & \text { if } i>j
\end{array},\right.
$$

where $a_{i}$ and $r_{i}$ are some constants. We thus define

$$
\Theta^{ \pm}=\left(\begin{array}{ccccc}
\mp \pi & \theta_{1}-\theta_{2}+\pi & \cdots & \theta_{1}-\theta_{L-1}+\pi & \theta_{1}-\theta_{L}+\pi \\
\theta_{2}-\theta_{1}-\pi & \mp \pi & \theta_{2}-\theta_{3}+\pi & \cdots & \theta_{2}-\theta_{L}+\pi \\
\vdots & \ddots & \ddots & \ddots & \vdots \\
\theta_{L-1}-\theta_{1}-\pi & \cdots & \theta_{L-1}-\theta_{L-2}-\pi & \mp \pi & \theta_{L-1}-\theta_{L}+\pi \\
\theta_{L}-\theta_{1}-\pi & \theta_{L}-\theta_{2}-\pi & \cdots & \theta_{L}-\theta_{L-1}-\pi & \mp \pi
\end{array}\right) .
$$

Let $U_{i}=\boldsymbol{e}_{\mathrm{L}}^{\top} \boldsymbol{U}_{\Omega_{i}}$ denote the value of $\boldsymbol{U}_{\Omega_{i}}$ at $\xi$, and we note that $r_{i}=-\frac{U_{i}}{2 \pi \mathrm{i}}$. Therefore, if we can show that

$$
S=\sum_{i=1}^{L} r_{i}
$$

is zero, the zero sum condition at $\xi$ follows.

Let $o_{i}$ denote the index of the collocation point in $\boldsymbol{x}$ corresponding to $\xi$ along $\Omega_{i}$. For the vector $\boldsymbol{r}=\left(r_{1}, \ldots, r_{L}\right)^{\top}$, We can write the $L$ computed values corresponding to the limit of the Cauchy transform along each $\Omega_{i}$ as

$$
\boldsymbol{\Phi}^{ \pm}=\left(\begin{array}{c}
\Phi_{1}^{ \pm} \\
\vdots \\
\Phi_{L}^{ \pm}
\end{array}\right)=\left(\begin{array}{c}
I \\
\vdots \\
I
\end{array}\right)+\left(\begin{array}{c}
\boldsymbol{e}_{o_{1}}^{\top} \\
\vdots \\
\boldsymbol{e}_{o_{L}}^{\top}
\end{array}\right) C^{ \pm} \boldsymbol{U}=\left(\begin{array}{c}
D \\
\vdots \\
D
\end{array}\right)+\Theta^{ \pm} \boldsymbol{r} .
$$

The constant $D$ consists of contributions which are independent of the angle at which $\xi$ is approached: the contributions of $\boldsymbol{U}$ from curves not in $\left\{\Omega_{1}, \ldots, \Omega_{L}\right\}$, as well as the constants $a_{i}$. Since

$$
\boldsymbol{e}_{i}^{\top} \Theta^{+}=\boldsymbol{e}_{i+1}^{\top} \Theta^{-}+\theta_{i+1}-\theta_{i}, \quad \text { (here we identify } L+1 \text { with } 1 \text { ) }
$$

we have

$$
\Phi_{i}^{+}=\Phi_{i+1}^{-}+\left(\theta_{i+1}-\theta_{i}\right) S
$$


Furthermore, the collocation system implies that

$$
\Phi_{i}^{+}=\Phi_{i}^{-} G_{i}
$$

Thus we get:

$$
\begin{aligned}
\Phi_{L}^{+} & =\Phi_{L}^{-} G_{L}=\Phi_{L-1}^{+} G_{L}+S\left[\left(\theta_{L-1}-\theta_{L}\right) G_{L}\right] \\
& =\Phi_{L-1}^{-} G_{L-1} G_{L}+S\left[\left(\theta_{L-1}-\theta_{L}\right) G_{L}\right] \\
& =\Phi_{L-2}^{+} G_{L-1} G_{L}+S\left[\left(\theta_{L-2}-\theta_{L-1}\right) G_{L-1} G_{L}+\left(\theta_{L-1}-\theta_{L}\right) G_{L}\right] \\
& \quad \vdots \\
& =\Phi_{L}^{+} G_{1} \cdots G_{L}+S \sum_{i=2}^{L}\left(\theta_{i-1}-\theta_{i}\right) G_{i} \cdots G_{L} .
\end{aligned}
$$

The well-posedness of the $\mathrm{RH}$ problem requires that:

$$
G_{1} \cdots G_{L}=I
$$

Hence we obtain

$$
0=S\left[\left(\theta_{L}-\theta_{1}\right) I+\sum_{i=2}^{L}\left(\theta_{i-1}-\theta_{i}\right) G_{i} \cdots G_{L}\right]
$$

By assumption the term in brackets is nonsingular, which implies that $S=0$ and the zero sum condition at $\xi$ follows. Applying this to each endpoint $\xi$ proves the theorem.

Q.E.D.

This theorem could potentially have consequences for the analytical solution: what it states is that, generically, we need not impose that the solution to the RH problem is bounded at the junction points, since it follows from the nonsingular junction condition.

The nonsingular junction condition is independent of the well-posedness of the RH problem, so if we are unfortunate we can find ourselves in a situation where it is not satisfied. Indeed, in [14], Stokes' multipliers to the Painlevé II transcendent were chosen particularly so that this condition was not satisfied. What was noted in this case was that the collocation system itself became singular: it had multiple solutions. Therefore, the zero sum condition could be appended to the linear system, to enforce that the solution chosen satisfied the zero sum condition. The following corollary states that this phenomena is true in general:

Corollary 6.5 Suppose the nonsingular junction condition is not satisfied at a junction $\xi$. Using the notation of the previous theorem, consider the collocation system with the condition

$$
\Phi_{L}^{+}=\Phi_{L}^{-} G_{L}
$$


replaced with

$$
\sum_{i=1}^{L} p_{i} U_{i}=0
$$

If the resulting system is nonsingular, then $\Phi_{L}^{+}=\Phi_{L}^{-} G_{L}$ is still satisfied. Since the computed solution $\boldsymbol{U}$ satisfies the zero sum condition, Lemma 6.2 applies.

Proof:

Consider (6.1). We cannot begin with $\Phi_{L}^{+}$; we do not know a priori that $\Phi_{L}^{+}=\Phi_{L}^{-} G_{L}$. However, the remaining equalities still hold, and we have

$$
\Phi_{L}^{-} G_{L}=\Phi_{L}^{+}+S \sum_{k=1}^{L}\left(\theta_{k-1}-\theta_{k}\right) G_{k} \cdots G_{L}=\Phi_{L}^{+},
$$

since $S=-\frac{1}{2 \pi \mathrm{i}} \sum U_{i}=0$.

Q.E.D.

\section{Examples}

We apply this numerical approach to two examples, taken directly from [8], though we make the construction of the $\mathrm{RH}$ problems explicit. We have also fixed several typos from [8]. In a way this helps to demonstrate another important aspect of having a numerical approach: it helps confirm (and in this case, correct) analytic formulæ.

\section{Painlevé III}

Our first example is the Painlevé III RH problem, for solving the Painlevé III differential equation:

$$
\frac{\mathrm{d}^{2} u}{\mathrm{~d} x^{2}}=\frac{1}{u}\left(\frac{d u}{\mathrm{~d} x}\right)^{2}-\frac{1}{x} \frac{d u}{\mathrm{~d} x}+\frac{4}{x}\left(\Theta_{0} u^{2}+1-\Theta_{\infty}\right)+4 u^{3}-\frac{4}{u} .
$$

Related to $u$ is the variable $y$, which satisfies

$$
\frac{\mathrm{d} y}{\mathrm{~d} x}=\frac{\Theta_{\infty}}{x} y+\frac{2 y}{u}
$$

What follows is the construction of a $\mathrm{RH}$ problem $\Phi^{+}(x ; z)=\Phi^{-}(x ; z) G(x ; z)$ so that $y$ (and thence $u$ ) is determined from $\Phi$. We restrict our attention to the case where $x$ is positive and real for simplicity: the curve $\Gamma$ of the RH problem depends on the phase of $x$.

Rather than specifying initial conditions, we specify Stokes' multipliers $s_{1}^{(0)}, s_{2}^{(0)}, s_{1}^{(\infty)}$ and $s_{2}^{(\infty)}$, which satisfy the equation

$$
2 \cos \pi \Theta_{0}+s_{1}^{(0)} s_{2}^{(0)} \mathrm{e}^{-\mathrm{i} \pi \Theta_{0}}=2 \cos \pi \Theta_{\infty}+s_{1}^{(\infty)} s_{2}^{(\infty)} \mathrm{e}^{\mathrm{i} \pi \Theta_{\infty}} .
$$


(In [8], two conflicting versions of this equation are stated. The equation presented here is indeed the correct one.) This equation ensures that the $\mathrm{RH}$ problem that is constructed is well-posed.

Often in physical applications it is the Stokes' multipliers which are known. What follows can be considered as a numerical map from Stokes' multipliers to initial conditions. Moreover, this map could potentially be inverted to map initial conditions to Stokes' multipliers. Since the Stokes' multipliers determine the asymptotic behaviour of the solution $u$, this could be used to connect asymptotic behaviour to initial conditions.

In this example, we choose (arbitrarily)

$$
\begin{aligned}
\Theta_{0} & =3.43, \quad \Theta_{\infty}=1.123 \\
s_{1}^{(0)} & =1, \quad s_{2}^{(0)}=2, \quad s_{1}^{(\infty)}=3, \\
s_{2}^{(\infty)} & =(-1)^{8} \cdot 877^{2} \frac{2}{3}\left((-1)^{.57}+\cos .123 \pi-\sin .07 \pi\right) \\
& =-0.5477638340114221 \ldots-0.4795328603546345 \ldots \text { i. }
\end{aligned}
$$

To construct the jump curves, we need a matrix $E$ that satisfies

$$
\begin{aligned}
\left(\begin{array}{cc}
1+s_{1}^{(\infty)} s_{2}^{(\infty)} & s_{1}^{(\infty)} \\
s_{2}^{(\infty)} & 1
\end{array}\right) & \left(\begin{array}{cc}
\mathrm{e}^{\mathrm{i} \pi \Theta_{\infty}} & \\
\mathrm{e}^{-\mathrm{i} \pi \Theta_{\infty}}
\end{array}\right) \\
& =E^{-1}\left(\begin{array}{cc}
1+s_{1}^{(0)} s_{2}^{(0)} & s_{1}^{(0)} \\
s_{2}^{(0)} & 1
\end{array}\right)\left(\begin{array}{cc}
\mathrm{e}^{-\mathrm{i} \pi \Theta_{0}} & \\
& \mathrm{e}^{\mathrm{i} \pi \Theta_{0}}
\end{array}\right) E
\end{aligned}
$$

Thus $E$ can be viewed as a generalized eigenvector matrix. One choice (found symbolically) is

$$
E=\left(\begin{array}{cc}
0.966469141021 \ldots+1.068382000028 \ldots \mathrm{i} & 0.778294876111 \ldots-0.163709626483 \ldots \mathrm{i} \\
1 & 1
\end{array}\right)
$$

We will also need to use a power function with a nonstandard branch cut. We define the power function with branch cut along angle $t$ as

$$
z^{y, t}=\left(\mathrm{e}^{\mathrm{i}(-\pi-t)} z\right)^{y} \mathrm{e}^{-\mathrm{i}(-\pi-t) y}
$$

We can now construct the RH problem. The left graph of Figure 2 depicts the jump curve $\Gamma$, along which we define the jump curves $G_{i, j}$ : for the function $\theta(x ; z)=\frac{\mathrm{i} x}{2}\left(z-\frac{1}{z}\right)$ 

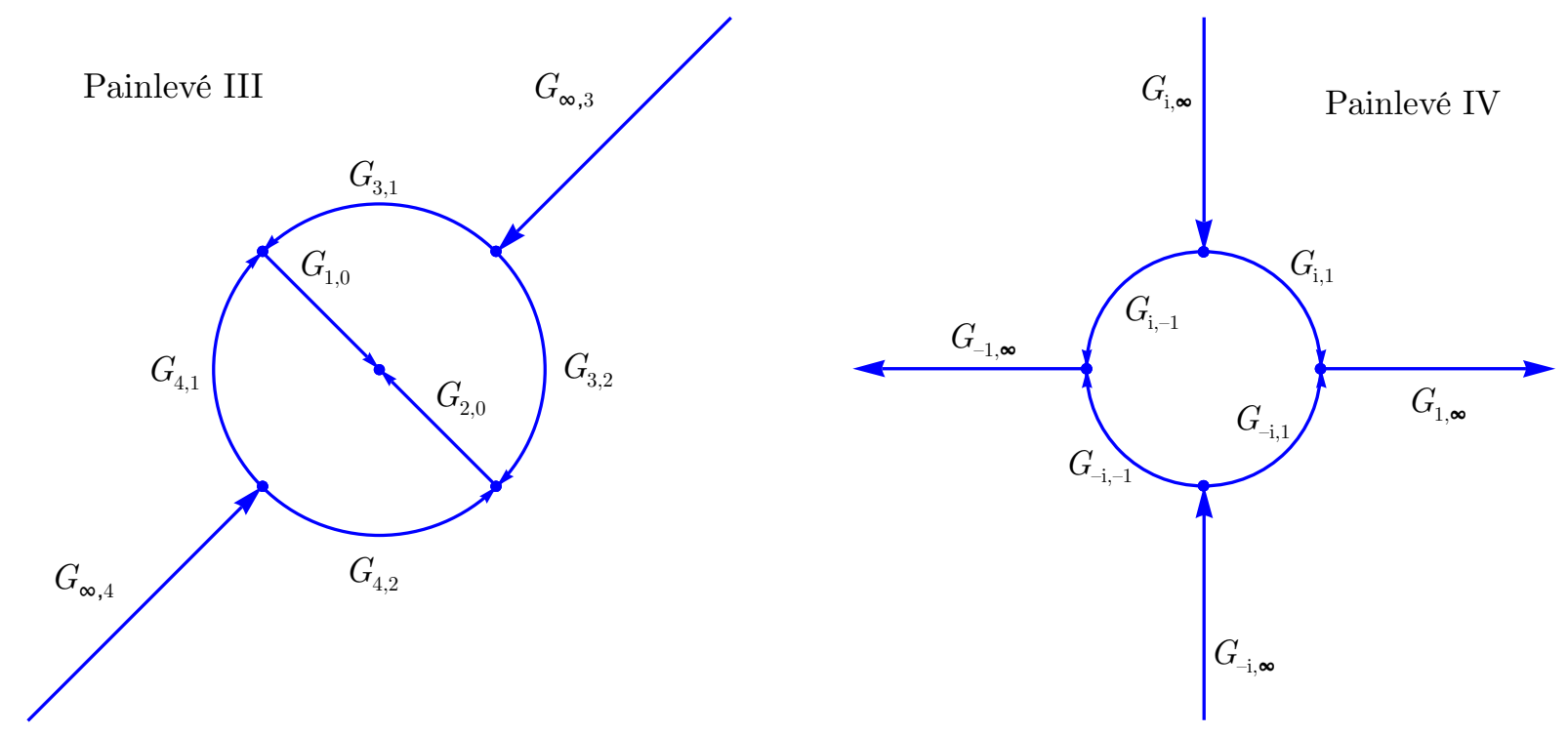

Figure 2: The jump curves for the Painlevé III (with junction points at $0, \mathrm{e}^{-5 \mathrm{i} \pi / 4}, \mathrm{e}^{-\mathrm{i} \pi / 4}, \mathrm{e}^{\mathrm{i} \pi / 4}$, $\mathrm{e}^{5 \mathrm{i} \pi / 4}$ and $\infty$ ) and Painlevé IV (with junction points at $-1,-\mathrm{i}, 1, \mathrm{i}$ and $\infty$ ) RH problems.

we have

$$
\begin{aligned}
& G_{3,2}=\left(\begin{array}{ll}
\mathrm{e}^{\theta} z^{\Theta_{0} / 2} & \\
& \mathrm{e}^{-\theta} z^{-\Theta_{0} / 2}
\end{array}\right) E\left(\begin{array}{ll}
z^{\Theta_{\infty} / 2} \mathrm{e}^{-\theta} & \\
& z^{-\Theta_{\infty} / 2} \mathrm{e}^{\theta}
\end{array}\right), \\
& G_{\infty, 3}=\left(\begin{array}{cc}
1 & -s_{1}^{(\infty)} \mathrm{e}^{2 \theta} z^{-\Theta_{\infty}} \\
0 & 1
\end{array}\right), \quad G_{4,0}=\left(\begin{array}{cc}
1 & -s_{1}^{(0)} \mathrm{e}^{2 \theta} z^{\Theta_{0}} \\
0 & 1
\end{array}\right), \\
& G_{1,4}=\left(\begin{array}{cc}
\mathrm{e}^{\theta} z^{\Theta_{0} / 2,-\pi / 2} & -s_{1}^{(0)} \mathrm{e}^{\theta} z^{\Theta_{0} / 2,-\pi / 2} \\
& \mathrm{e}^{-\theta} z^{-\Theta_{0} / 2,-\pi / 2}
\end{array}\right) E\left(\begin{array}{cc}
z^{\Theta \infty / 2,-\pi / 2} \mathrm{e}^{-\theta} & s_{1}^{(\infty)} z^{-\Theta_{\infty} / 2,-\pi / 2} \mathrm{e}^{\theta} \\
& z^{-\Theta_{\infty} / 2,-\pi / 2} \mathrm{e}^{\theta}
\end{array}\right), \\
& G_{1,2}=\left(\begin{array}{cc}
\mathrm{e}^{\theta} z^{-\Theta_{\infty} / 2} & \\
& \mathrm{e}^{-\theta} z^{\Theta_{\infty} / 2}
\end{array}\right) E^{-1}\left(\begin{array}{cc}
z^{-\Theta_{0} / 2,0} \mathrm{e}^{\mathrm{i} \pi \Theta_{0}-\theta} \\
-s_{2}^{(0)} z^{-\Theta_{0} / 2,0} \mathrm{e}^{-\mathrm{i} \pi \Theta_{0}-\theta} & z^{\Theta_{0} / 2,0} \mathrm{e}^{\theta-\mathrm{i} \pi \Theta_{0}}
\end{array}\right), \\
& G_{3,4}=\left(\begin{array}{cc}
\mathrm{e}^{\theta} z^{-\Theta_{\infty} / 2} & -s_{1}^{(\infty)} \mathrm{e}^{\theta} z^{-\Theta_{\infty} / 2} \\
& \mathrm{e}^{-\theta} z^{\Theta} / 2
\end{array}\right) E^{-1}\left(\begin{array}{ll}
z^{-\Theta_{0} / 2} \mathrm{e}^{-\theta} & \\
& z^{\Theta_{0} / 2} \mathrm{e}^{\theta}
\end{array}\right),
\end{aligned}
$$

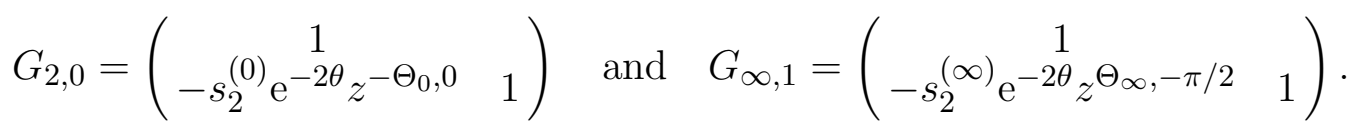

Along each curve, we need to decide at how many points to sample. We choose this adaptively: we take sufficiently many points so that the omitted terms of the Chebyshev expansion are each less than a given tolerance.

We can now apply our numerical approach to determine an approximation $\tilde{\Phi}$. However, we are really interested in the solution $u$ to the Painlevé III ODE. We have the following 
definition:

$$
y(x)=-\mathrm{i} x \lim _{z \rightarrow \infty} z \Phi_{12}(x ; z)=-\mathrm{i} x \lim _{z \rightarrow \infty} z[\mathcal{C} U(x ; z)]_{12},
$$

where 12 denotes the $(1,2)$ entry of the matrix. (In [8], this was mistakenly given as a definition for $u(x)$.) But the limit of the Cauchy transform is simply an integral of the function:

$$
\lim _{z \rightarrow \infty} z \mathcal{C} U(z)=\frac{1}{2 \pi \mathrm{i}} \int_{\Gamma} \frac{z}{t-z} U(t) \mathrm{d} t=-\frac{1}{2 \pi \mathrm{i}} \int_{\Gamma} U(t) \mathrm{d} t .
$$

Moreover, we can evaluate this integral by transforming to the unit interval

$$
\int_{\Gamma} U(t) \mathrm{d} t=\sum_{i} \int_{\Gamma_{i}} U_{i}(t) \mathrm{d} t=\sum_{i} \int_{-1}^{1} \frac{U_{i}\left(M_{i}^{-1}(p)\right)}{M^{\prime}\left(M_{i}^{-1}(p)\right)} \mathrm{d} p .
$$

Each of these integrals can be evaluated with Clenshaw-Curtis quadrature [3]. This quadrature routine computes an integral over $\mathbb{I}$ in $\mathcal{O}(n \log n)$ time using the value of the integrand at the nodes $\boldsymbol{x}^{\mathbb{I}}$. In our case, the values are simply $\boldsymbol{U}_{i}$, with a zero appended in the case that $\Gamma_{i}$ is unbounded, multiplied entrywise by $M^{\prime}\left(M_{i}^{-1}\left(\boldsymbol{x}^{\mathbb{I}}\right)\right)^{-1}$. We define this approximation as $\mathcal{Q}_{\Gamma_{i}} \boldsymbol{U}_{i}$. We thus obtain the approximation

$$
\int_{\Gamma} U(t) \mathrm{d} t \approx \mathcal{Q} \boldsymbol{U}=\sum \mathcal{Q}_{\Gamma_{i}} \boldsymbol{U}_{i}
$$

and hence

$$
y(x) \approx \frac{x}{2 \pi} \mathcal{Q U} .
$$

Now to find $u$, we will use the definition

$$
u=\frac{2 x y}{x y_{x}-\Theta_{\infty} y} .
$$

However, to obtain $u$, we need to compute $y_{x}$ at $x$. Fortunately, derivatives commute with the limit, hence

$$
y_{x}(x)=-\mathrm{i} \lim _{z \rightarrow \infty} z \Phi_{12}(x ; z)-\mathrm{i} x \lim _{z \rightarrow \infty} z \Phi_{x, 12}(x ; z) .
$$

We already have computed $\Phi_{12}$, but we now also need the derivative of $\Phi$ with respect to $x$. This is straightforward by simply differentiating the RH problem:

$$
\Phi_{x}^{+}-\Phi_{x}^{-} G=\Phi^{-} G_{x} \quad \text { and } \quad \Phi_{x}(\infty)=0 .
$$

Replacing $\Phi_{x}$ by $\mathcal{C} U_{x}$, we see that this equation is of exactly the same form as (2.1), with only a different right hand side. Therefore, having constructed $\boldsymbol{U}$, we can determine a vector $\boldsymbol{U}_{x}$ by reusing the matrix of (2.3) (hence all the computed Cauchy matrices) to solve

$$
\left[C^{+}-\operatorname{rdiag}(\boldsymbol{G}) C^{-}\right] \boldsymbol{U}_{x}=\operatorname{rdiag}\left(G_{x}(\boldsymbol{x})\right)\left[\left(\begin{array}{c}
I \\
\vdots \\
I
\end{array}\right)+C^{-} \boldsymbol{U}\right] .
$$



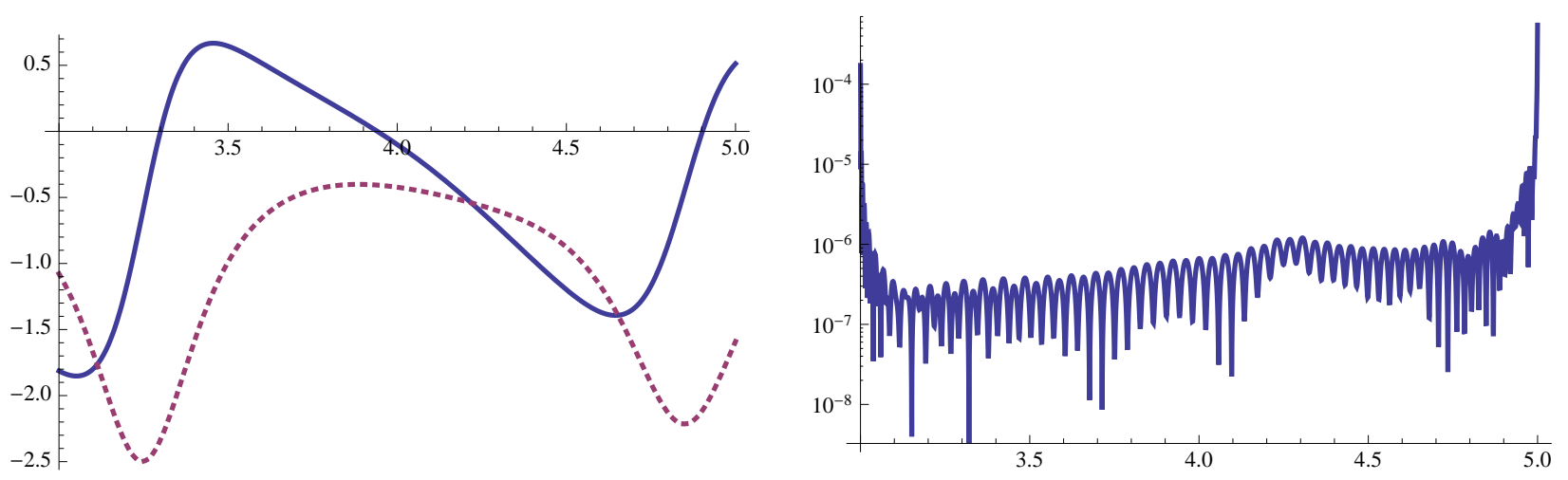

Figure 3: The real (solid line) and imaginary (dotted line) parts of a solution to Painlevé III on the left, with its relative error in residual on the right.

Thus we obtain

$$
u(x) \approx \frac{2 x[\mathcal{Q U}]_{12}}{x\left[\mathcal{Q} \boldsymbol{U}_{x}\right]_{12}+\left(1-\Theta_{\infty}\right)[\mathcal{Q U}]_{12}} .
$$

In Figure 3, we plot the computed solution, and demonstrate that it does satisfy the Painlevé III ODE by finding the error in residual, computed by evaluating the approximation at 85 mapped Chebyshev points between 3 and 5. In this case seven digits of accuracy are about how many can be expected: the last Chebyshev coefficient is on the order of $10^{-10}$ and two derivatives should multiply the error by about $85^{2} \approx 10^{4}$.

Perhaps a more useful calculation would be the Sine-Gordon reduction to Painlevé III. This has a significantly simpler RH formulation, consisting of four rays emanating from the origin. We instead chose the more general Painlevé III RH problem, to demonstrate the flexibility of the framework.

\section{Painlevé IV}

We now consider the Painlevé IV RH problem, for computing the solutions to the Painlevé IV ODE:

$$
\frac{\mathrm{d}^{2} u}{\mathrm{~d} x^{2}}=\frac{1}{2 u}\left(\frac{\mathrm{d} u}{\mathrm{~d} x}\right)^{2}+\frac{3}{2} u^{3}+4 x u^{2}+\left(2+2 x^{2}-4 \Theta_{\infty}\right) u-\frac{8 \Theta^{2}}{u},
$$

where $\Theta_{\infty}$ and $\Theta$ are constants. Solutions are specified by the four Stokes' multipliers $s_{1}, s_{2}, s_{3}$ and $s_{4}$, satisfying the relationship

$$
\left(1+s_{2} s_{3}\right) \mathrm{e}^{2 \mathrm{i} \pi \Theta_{\infty}}+\left[s_{1} s_{4}+\left(1+s_{3} s_{4}\right)\left(1+s_{1} s_{2}\right)\right] \mathrm{e}^{-2 \mathrm{i} \pi \Theta_{\infty}}=2 \cos 2 \pi \Theta .
$$

Again, we choose these constants arbitrarily:

$$
\begin{aligned}
\Theta & =3.43, \quad \Theta_{\infty}=1.123 \\
s_{1} & =1, \quad s_{2}=2, \quad s_{3}=\frac{1}{3}, \\
s_{4} & =-2.1687404438883131 \ldots-1.4647891159376507 \ldots i .
\end{aligned}
$$



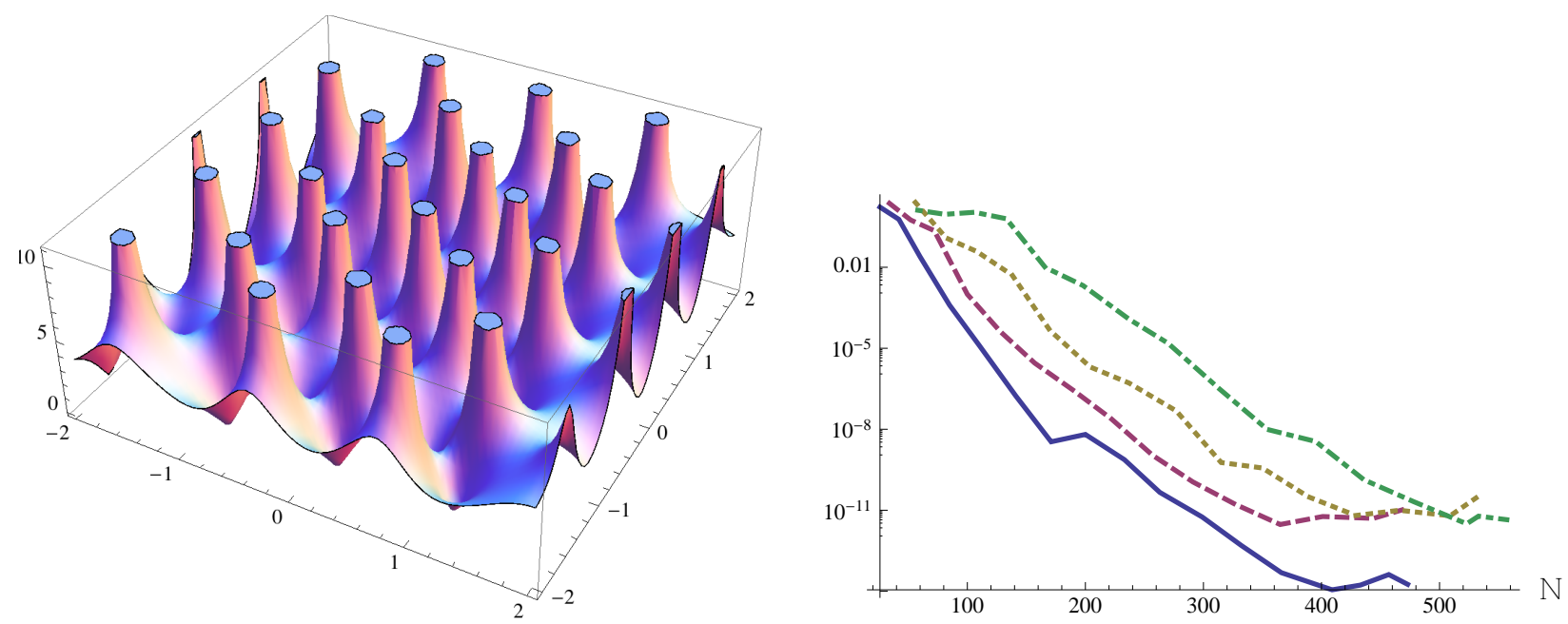

Figure 4: On the left, a plot of the absolute value of a solution to Painlevé IV in the complex plane. On the right, the relative error of the approximation for $x=0$ (solid), 1 (dashed), 2 (dotted) and 3 (dash and dotted).

We also need a matrix $E$, but in this case it is simply an eigenvector matrix (where we happen to know the eigenvalues)

$$
\left(\begin{array}{cc}
1 & \\
s_{1} & 1
\end{array}\right)\left(\begin{array}{cc}
1 & s_{2} \\
& 1
\end{array}\right)\left(\begin{array}{cc}
1 & \\
s_{3} & 1
\end{array}\right)\left(\begin{array}{cc}
1 & s_{4} \\
& 1
\end{array}\right)\left(\begin{array}{ll}
\mathrm{e}^{2 \mathrm{i} \pi \Theta_{\infty}} & \\
& \mathrm{e}^{2 \mathrm{i} \pi \Theta_{\infty}}
\end{array}\right)=E^{-1}\left(\begin{array}{ll}
\mathrm{e}^{-2 \mathrm{i} \pi \Theta} & \\
& \mathrm{e}^{2 \mathrm{i} \pi \Theta}
\end{array}\right) E
$$

The right graph of Figure 2 depicts the jump curve $\Gamma$, along which we define the jump curves $G_{i, j}$ for $\theta(x ; z)=\frac{z^{2}}{2}+x z$ :

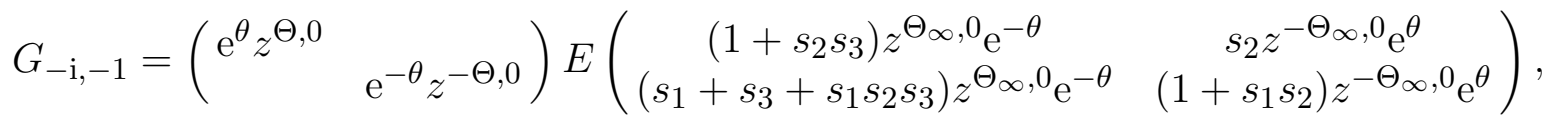

$$
\begin{aligned}
& G_{-\mathrm{i}, 1}=\left(\begin{array}{cc}
\mathrm{e}^{\theta} z^{-\Theta \infty} & \\
& \left.\mathrm{e}^{-\theta} z^{\Theta}\right)_{\infty}
\end{array}\right) E^{-1}\left(\begin{array}{ll}
z^{-\Theta} \mathrm{e}^{-\theta} & \\
& z^{\Theta} \mathrm{e}^{\theta}
\end{array}\right), \\
& G_{\mathrm{i}, 1}=\left(\begin{array}{cc}
\mathrm{e}^{\theta} z^{\Theta} & \\
& \mathrm{e}^{-\theta} z^{-\Theta}
\end{array}\right) E\left(\begin{array}{cc}
z^{\Theta \infty} \mathrm{e}^{-\theta} & \\
s_{1} z^{\Theta} \mathrm{e}^{-\theta} & z^{-\Theta \infty} \mathrm{e}^{\theta}
\end{array}\right) \\
& G_{\mathrm{i},-1}=\left(\begin{array}{cc}
\left(1+s_{1} s_{2}\right) \mathrm{e}^{\theta} z^{-\Theta_{\infty}, 0} & -s_{2} \mathrm{e}^{\theta} z^{-\Theta_{\infty}, 0} \\
-s_{1} \mathrm{e}^{-\theta} z_{0}^{\Theta} & \mathrm{e}^{-\theta} z^{\Theta \infty}, 0
\end{array}\right) E^{-1}\left(\begin{array}{cc}
z^{-\Theta, 0} \mathrm{e}^{-\theta} & \\
& z^{\Theta, 0} \mathrm{e}^{\theta}
\end{array}\right) \\
& G_{-1, \infty}=\left(\begin{array}{cc}
1 & \\
s_{3} \mathrm{e}^{-2 \theta} z^{2 \Theta_{\infty}, 0} & 1
\end{array}\right), \quad G_{\infty,-\mathrm{i}}=\left(\begin{array}{cc}
1 & -s_{4} \mathrm{e}^{2 \theta} z^{-2 \Theta_{\infty}, 0} \\
1
\end{array}\right), \\
& G_{1, \infty}=\left(\begin{array}{cc}
1 & \\
s_{1} \mathrm{e}^{-2 \theta} z^{2 \Theta_{\infty}} & 1
\end{array}\right) \quad \text { and } \quad G_{\infty, \mathrm{i}}=\left(\begin{array}{cc}
1 & -s_{2} \mathrm{e}^{2 \theta} z^{-2 \Theta_{\infty}} \\
1
\end{array}\right) .
\end{aligned}
$$

Again, we have everything in place for our numerical approach, choosing the number of 
collocation points on each curve adaptively. The relation between $\Phi$ and $u$ is

$$
u(x)=-2 x-\lim _{z \rightarrow \infty} \partial_{x} \log \Phi_{12}(x ; z)=-2 x-\lim _{z \rightarrow \infty} \frac{\Phi_{x, 12}(x ; z)}{\Phi_{12}(x ; z)} .
$$

Thus we define

$$
u(x) \approx-2 x-\frac{\left[\mathcal{Q} \boldsymbol{U}_{x}\right]_{12}}{[\mathcal{Q U}]_{12}} .
$$

In the left graph of Figure 4, we plot the absolute value of a solution to Painlevé IV in the complex plane. Note the presence of poles. This is one aspect where our method outperforms a standard ODE solver; integrating past poles is not an issue, since $x$ is merely a parameter. At the pole, the RH problem does not have a solution. This manifests itself in our numerical approach by a badly condition collocation system.

In the right graph of Figure 4, we compare the convergence of the approximation as $N$ (the total number of collocation points) increases, where the distribution of number of points on each curve is chosen adaptively. We compare these approximations with the approximation found with a much larger value of $N$. Spectral convergence and stability of the method is clear, as is the slower convergence rate as $x$ becomes large.

\section{Conclusions and future work}

We have constructed a framework for computing RH problems, and demonstrated its effectiveness by applying it to the computation of Painlevé transcendents. The world of RH problems is large, and this opens up a new numerical technique applicable to fields from integrable systems to orthogonal polynomials and random matrix theory. It is our belief that many problems exist in these fields which, while at first seemingly intractable from a numerical perspective, can now be efficiently and reliably computed.

We have provided an implementation of this framework in MATHEMATICA in RHPackage [13], including the implementation of the two examples from this paper. The only piece that cannot be translated to an alternate programming language in a straightforward manner is the computation of hypergeometric functions used as the seed to Algorithm 5.2, as built-in routines for hypergeometric functions in, say, MATLAB are not reliable [16]. However, there are many methods for computing hypergeometric functions [12], and it is possible that one of these is accurate and reliable in the regime we are interested in.

What makes $\mathrm{RH}$ problems so powerful in an analytic context is nonlinear steepest descent; the problem can be deformed so that oscillations (as seen in our two examples) become exponential decay. While our numerical approach breaks down in the two examples as $x$ becomes large, using the deformed contour will allow high accuracy in the asymptotic regime. However, the resulting paths of steepest descent are not trivially deformable to the unit interval, hence the framework constructed cannot be directly applied. However, errors in the 
path of steepest descent do not result in errors in the approximation, and thus the path can be replaced by a piecewise affine interpolant.

An example application of this is the Hastings-McLeod solution [9] to Painlevé II, which has applications in random matrix theory via the Tracy-Widom distribution [17]. Perturbations of the initial conditions result in either high oscillations or poles, neither of which is present in the true solution. In [14], we demonstrated that it can be computed near the origin using the current framework; or, alternatively (and more efficiently), using the Fredholm determinant representation [2]. Both approaches break down as $x$ becomes large; however, using the deformed contour will result in a reliable numerical method for all $x$. This will be the topic of a future paper.

This framework has been based on the Chebyshev basis. Another possible basis are the Legendre polynomials, whose Cauchy transforms decay to high asymptotic order due to orthogonality:

$$
\mathcal{C} P_{k}(z)=\frac{1}{2 \pi \mathrm{i}} \int_{-1}^{1} \frac{P_{k}(x)}{x-z} \mathrm{~d} x=\frac{1}{2 \pi \mathrm{i} z^{k}} \int_{-1}^{1} \frac{P_{k}(x) x^{k}}{x-z} \mathrm{~d} x=\mathcal{O}\left(z^{-k-1}\right) .
$$

This could lead to sparser matrices, and, potentially, a faster solution of the collocation system. Moreover, an explicit formula is known:

$$
\mathcal{C} P_{k}(z)=-\frac{2^{k+1}(k !)^{2}}{2 \pi \mathrm{i}(2 k+1) !(z-1)^{k+1}}{ }_{2} F_{1}\left(\begin{array}{c}
k+1, k+1 \\
2 k+2
\end{array} ; \frac{2}{1-z}\right) .
$$

However, we have used the Chebyshev basis in this paper due to the availability of a fast and accurate transform between a set of nodes which include the endpoints and the series' coefficients.

One last issue that must be resolved is a proof of convergence. Assuming the RH problem has a solution, it is our feeling that convergence of the numerical approximation should follow, since $\mathcal{C}^{+} U-\left[\mathcal{C}^{-} U\right] G$ is a bounded linear operator from the space of Hölder-continuous functions $U$ defined on $\Gamma$ which satisfy (the infinite dimensional version of) the zero sum condition to the space of Hölder-continuous functions $U$ defined on $\Gamma$. Indeed, all numerical experiments have shown clear and stable convergence, depending only on the condition number of the collocation system. However, since the domain space and range space of this operator differ, the standard convergence proofs for collocation methods do not apply.

Acknowledgments: I thank Alex Barnett, discussions with whom lead me to Algorithm 5.2, Andrew Dienstfrey, who pointed me to his thesis, and Andre Weideman, who recommended replacing Lerch transcendents with hypergeometric functions. 


\section{References}

[1] Ablowitz, M.J. and Segur, H., Solitons and the Inverse Scattering Transform, SIAM, 2006.

[2] Bornemann, F., On the numerical evaluation of Fredholm determinants, Maths Comp 79 (2010), 871-915.

[3] Clenshaw, C. W. and Curtis, A. R., A method for numerical integration on an automatic computer, Numer. Math. 2 (1960), 197-205.

[4] Deift, P., Orthogonal Polynomials and Random Matrices: a Riemann-Hilbert Approach, AMS, 2000.

[5] Deift, P. and Zhou, X., A steepest descent method for oscillatory Riemann-Hilbert problems, Bulletin AMS 26 (1992), 119-124.

[6] Dienstfrey, A., The Numerical Solution of a Riemann-Hilbert Problem Related to Random Matrices and the Painlevé V ODE, Ph.D. Thesis, Courant Institute of Mathematical Sciences, 1998.

[7] Elliott, D., Uniform asymptotic expansions of the Jacobi polynomials and an associated function, Maths Comp. 25 (1971), 309-315.

[8] Fokas, A.S., Its, A.R., Kapaev, A.A. and Novokshenov,V.Y., Painlevé transcendents: the Riemann-Hilbert approach, AMS, 2006.

[9] Hastings, SP and McLeod, JB, A boundary value problem associated with the second Painlevé transcendent and the Korteweg-de Vries equation, Archive for Rational Mechanics and Analysis 73 (1980), 31-51.

[10] Muskhelishvili, N.I., Singular Integral Equations, Groningen: Noordhoff (based on the second Russian edition published in 1946), 1953.

[11] Nasser, M.M.S., Numerical solution of the Riemann-Hilbert problem, Punjab University Journal of Mathematics 40 (2008), 9-29.

[12] Olver, F. W. J., Lozier, D. W., Boisvert, R. F. and Clark, C. W., NIST Handbook of Mathematical Functions, Cambridge University Press, 2010.

[13] Olver, S., RHPackage, http://www.comlab.ox.ac.uk/people/ Sheehan.0lver/projects/RHPackage.html

[14] Olver, S., Numerical solution of Riemann-Hilbert problems: Painlevé II, preprint, NA-09/09, Maths Institute, Oxford University.

[15] Olver, S., Computing the Hilbert transform and its inverse, Maths Comp., to appear. 
[16] Pearson, J., Computation of Hypergeometric Functions, MSc. Thesis, University of Oxford, 2009.

[17] Tracy, C.A. and Widom, H., Level-spacing distributions and the Airy kernel, Comm. Math. Phys. 159 (1994), 151-174.

[18] Wegert, E., An iterative method for solving nonlinear Riemann-Hilbert problems, $J$. Comp. Appl. Maths 29 (1990), 327.

[19] Wegmann, R., Discrete Riemann-Hilbert problems, interpolation of simply closed curves, and numerical conformal mapping, J. Comp. Appl. Maths 23 (1988), $323-352$.

[20] Wegmann, R., An iterative method for the conformal mapping of doubly connected regions, J. Comp. Appl. Maths 14 (1986), 79-98. 